\title{
Application of a Weighted Average Surrogate Approach to Helicopter Rotor Blade Vibration Reduction
}

\author{
Bryan Glaz* \\ Department of Aerospace Engineering, The University of Michigan, Ann Arbor, MI, 48109, USA \\ Tushar Goel ${ }^{\dagger}$ \\ Department of Mechanical and Aerospace Engineering, The University of Florida, Gainesville, FL, 32611, USA \\ Li Liu ${ }^{\ddagger}$ and Peretz P. Friedmann ${ }^{\S}$ \\ Department of Aerospace Engineering, The University of Michigan, Ann Arbor, MI, 48109, USA \\ Raphael T. Haftka \\ Department of Mechanical and Aerospace Engineering, The University of Florida, Gainesville, FL, 32611, USA
}

\begin{abstract}
The advantages of employing multiple approximation methods and the effectiveness of weighted average surrogate modeling for approximation and reduction of helicopter vibrations is studied. Multiple surrogates, including the weighted average approach, are considered so that the need to identify the "best" approximation method for the rotor vibration reduction problem is eliminated. Various approximation methods are used to generate a vibration objective function corresponding to a flight condition in which bladevortex interaction causes high levels of vibration. The design variables consist of the crosssectional dimensions of the structural member of the blade and non-structural masses. The optimized designs are compared with a baseline design resembling an MBB BO-105 blade. The results indicate that (a) multiple surrogates can be used to locate low vibration designs which would be overlooked if only a single approximation method was employed, and (b) that the weighted average approach protects against the worst individual surrogate, while performing as well as the best individual surrogate. Furthermore, the surrogates were used in a global sensitivity analysis to identify the most significant design variables for the vibration reduction problem.
\end{abstract}

\section{Nomenclature}

$\begin{array}{ll}c & \text { Blade chord } \\ C_{W} & \text { Helicopter weight coefficient } \\ C_{d 0} & \text { Blade profile drag coefficient } \\ C_{d f} & \text { Flat plate drag coefficient } \\ \mathbf{D} & \text { Vector of design variables } \\ d_{1}, d_{2} & \text { User defined parameters used in weighted average surrogate construction } \\ \mathrm{E} & \text { Young's modulus } \\ E[] & \text { Expected value operator } \\ E_{i} & \text { Global error metric corresponding to the } i^{\text {th }} \text { surrogate } \\ E_{\text {avg }} & \text { Average of the global error metrics for all surrogates }\end{array}$

\footnotetext{
*Ph.D. Candidate, Student Member AIAA.

$\dagger$ Ph.D. Candidate, Student Member AIAA.

${ }^{\ddagger}$ Postdoctoral Researcher, Member AIAA

$\S$ François-Xavier Bagnoud Professor, Fellow AIAA, AHS

๑Distinguished Professor, Fellow AIAA.
} 


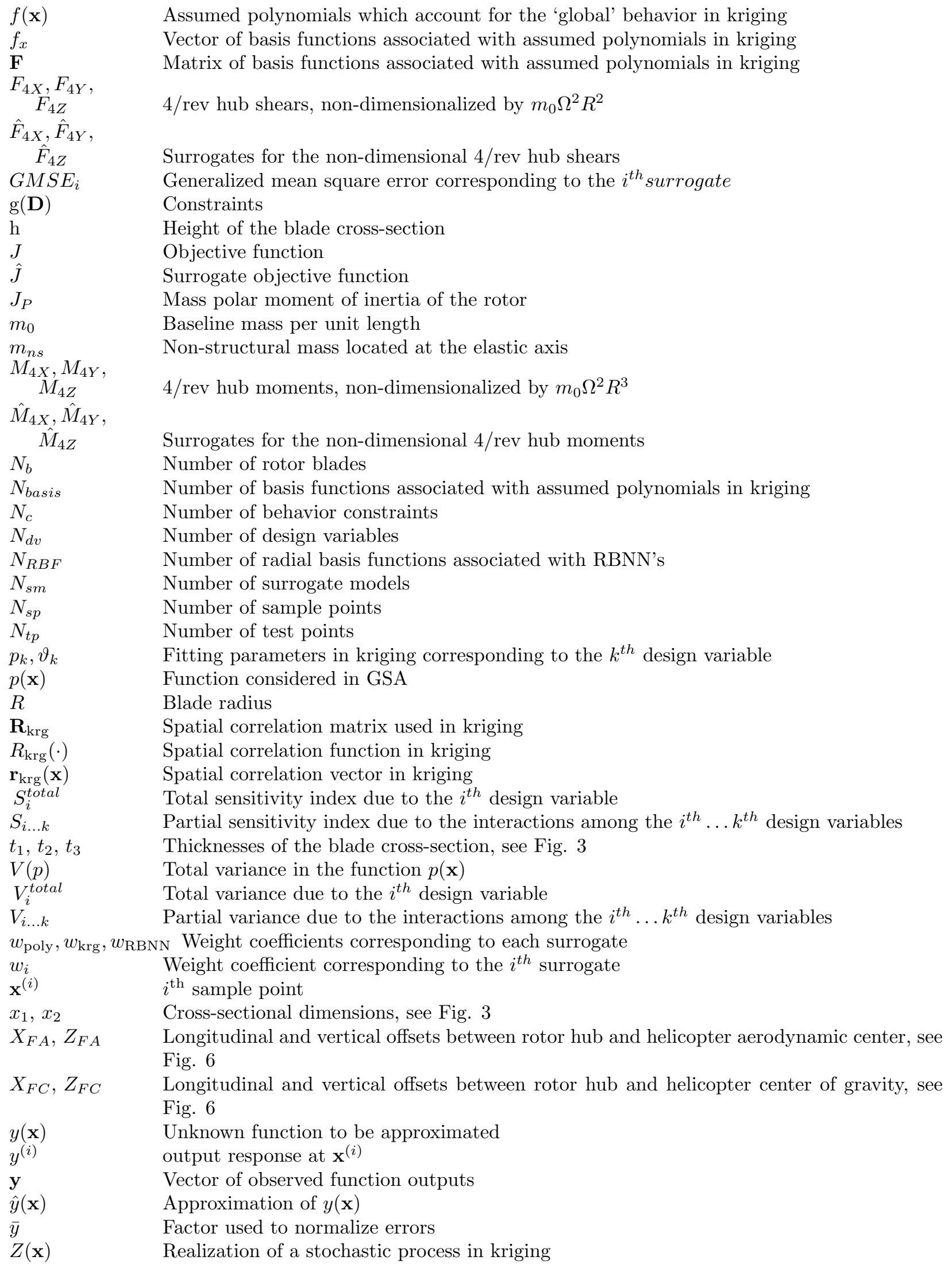

Symbols

$\alpha_{d}$

Flight descent angle, see Fig. 6 


$\begin{array}{ll}\alpha_{i} & \text { Weight associated with the } i^{\text {th }} \text { radial basis function in RBNN's } \\ \beta & \text { Vector of coefficients used in kriging } \\ \hat{\beta} & \text { Generalized least squares estimate of } \beta \\ \beta_{0}, \beta_{i}, \beta_{i j} & \text { Fitting coefficients in polynomial regression } \\ \beta_{p} & \text { Blade precone angle } \\ \epsilon_{\mathrm{pr}} & \text { Approximation error in polynomial regression } \\ \varepsilon & \text { Absolute percent error of surrogate predictions } \\ \lambda_{k} & \text { Hover stability eigenvalue for } k^{t h} \text { mode } \\ \zeta_{k}, \omega_{k} & \text { Real and imaginary parts of } \lambda_{k}, \text { respectively } \\ \mu & \text { Advance ratio } \\ \nu & \text { Poisson's ratio } \\ \Omega & \text { Rotor angular speed } \\ \omega_{F 1}, \omega_{L 1}, \omega_{T 1} & \text { Fundamental rotating flap, lead-lag and torsional frequencies, } / \mathrm{rev} \\ \omega_{L}, \omega_{U} & \text { Lower and upper bounds for frequency constraints, } / \text { rev } \\ \phi_{\mathrm{RBNN}} & \text { Radial basis functions used in RBNN's } \\ \rho_{\text {filler }} & \text { Material density for non-structural filler mass } \\ \rho_{\text {struct }} & \text { Material density for the structural member of the blade } \\ \sigma & \text { Rotor solidity } \\ \sigma_{\text {allowable }} & \text { Allowable blade stress } \\ \sigma_{x x}, \sigma_{x \eta}, \sigma_{x} \zeta & \text { Blade stresses } \\ \sigma_{v a r}^{2} & \text { Variance of the Gaussian process } Z(\mathbf{x}) \\ \hat{\sigma}_{v a r}^{2} & \text { Generalized least squares estimate of } \sigma_{v a r}^{2} \\ \sigma_{Y} & \text { Yield stresses } \\ \tau & \text { Parameter which control radius of influence for each neuron in RBNN's } \\ \theta_{\mathrm{pt}} & \text { Blade built-in pre-twist angle }\end{array}$

\section{Introduction}

$\mathrm{V}$

IBRATION is one of the most critical concerns in the design of modern rotorcraft. Stricter demands for enhanced performance, comfort, and customer acceptance require designs with reduced vibration levels. In helicopters, the dominant source of vibrations is the rotor, which transfers vibrations to the rotor hub and fuselage at harmonics that are predominantly integer multiple of $N_{b} / \mathrm{rev}$, where $N_{b}$ is the number of blades.

During the last 25 years, two principal approaches to vibration reduction have emerged. The first approach is passive and uses structural/multidisciplinary optimization for reducing vibrations. ${ }^{1-3}$ The second approach utilizes active control methods. ${ }^{4,5}$ The passive approach is used by blade designers to improve the vibration characteristics of the rotor. On the other hand, the active approach is still considered a research topic which is slowly approaching implementation. This study is aimed at the passive approach where the vibration reduction problem is formulated as a mathematical optimization problem subject to appropriate constraints. The objective function consists of a suitable combination of the $N_{b} /$ rev hub shears and moments that are computed from an aeroelastic response code; constraints are specified on blade stability margins, frequency, blade geometry, autorotational properties, and blade stresses. The design variables can be dimensions of the blade cross-section, mass and stiffness distributions along the span, or geometrical parameters which define advance geometry tips. Typical levels of vibration reduction achieved with passive approaches have been in the range of $30-60 \%$ relative to a baseline design.

Due to the complex rotary-wing aerodynamic environment, the aeroelastic response simulations needed for vibratory load calculations are computationally expensive. Therefore numerous evaluations of the vibration objective function are costly. Consequently, direct combination of the objective function generated by an aeroelastic response simulation with traditional optimization algorithms is computationally very expensive. Moreover, traditional optimization search algorithms can converge to local optima, which are known to occur in this class of problems.

To overcome these obstacles, approximation concepts have been used. ${ }^{6-8}$ A typical approach when using approximation, or surrogate methods, is to assess the performance of various surrogate models and then select the one which performs the best. This method was recently applied to the helicopter vibration reduction problem in Ref. 9, where $2^{\text {nd }}$ order polynomial response surfaces, radial basis function interpolation, and kriging were considered for construction of the vibratory hub load surrogates. The study was based 
on a comprehensive helicopter simulation code which utilizes advanced modeling techniques such as free wake modeling. Eight design variables were used to characterize the blade's spanwise mass and stiffness distributions, including blade cross-sectional dimensions and non-structural masses. The results showed that kriging was the most accurate global approximation method considered and led to the best design, which produced $67 \%$ vibration reduction.

Most research dealing with surrogate modeling has been concerned with the choice among different surrogates. However, the choice of the "best" surrogate model is determined by a number of factors, and once selected the choice of the "best" surrogate model is seldom reviewed. These factors include: the number of points used to construct the surrogate model (sampling density), the scheme used to select points (design of experiment), and parameters/nature of the surrogate model. Different surrogate models have been shown to perform well under different conditions and for different objectives. For instance, for a given application, the most accurate approximation method may not necessarily lead to the most optimal design. Thus, a single approximation method has not distinguished itself as the most suitable for engineering applications.

As an alternative to seeking the "best" approximation method, there has been recent work on the collaborative use of an ensemble of surrogates. ${ }^{10}$ Typically, the cost of obtaining data required for developing surrogates is high, so it is desirable to extract as much information as possible from the data. Since surrogates can be constructed without significant expense compared to the cost of acquiring data, use of an ensemble of surrogates can prove effective in distilling correct trends from the data while protecting against poor surrogate models. The combination of multiple surrogates is motivated by our inability to find a unique solution to the non-linear inverse problem of identifying the model from a limited set of data ${ }^{6}$ and essentially serves as an approach to account for uncertainty in the surrogates.

While the ensemble of surrogate approach has yet to be applied to the helicopter rotor blade vibration reduction problem, it has been used in various optimization applications. ${ }^{10-13}$ In Ref. 10, the generalized mean squared cross-validation error was used to develop a weighted average surrogate model. For a number of analytical and applied problems, it was demonstrated that the weighted average surrogate model protects against the worst individual surrogate in all cases while performing as well as the best.

In addition to replacing computationally expensive objective functions in optimization, the surrogates can also be used to replace expensive global sensitivity analysis (GSA) ${ }^{14}$ function evaluations. Global sensitivity analysis is used to rank the design variables in terms of influence on the objective function over the entire design space, as opposed to partial derivatives which estimate the local sensitivity of a function in the vicinity of a design point. The GSA based on Ref. 14 has been used in various applications, ${ }^{15-17}$ however identification of the most important design variables using surrogates and GSA has not been considered in previous helicopter vibration studies.

The overall objectives of this paper are to demonstrate the advantages of utilizing multiple surrogates, which results in little additional cost compared to using only one surrogate, and to apply GSA to the helicopter vibration reduction problem. The specific objectives of the paper are listed below.

- Develop the weighted average surrogate model based on three approximation methods: polynomial response surfaces, kriging, and radial basis neural networks.

- Demonstrate that the weighted surrogate approach always protects against the worst individual surrogate while generally performing as well as the best individual surrogate in terms of predictive capability and optimization.

- Locate multiple low vibration blade designs by optimizing the individual surrogates as well as the weighted average models.

- Perform surrogate based GSA to identify the most significant design variables for helicopter vibration reduction using structural optimization.

\section{Overview of the Aeroelastic Response and Stability Analysis}

The simulation code used in this study is based on a comprehensive aeroelastic analysis code. ${ }^{18-24}$ The aeroelastic response analysis can represent the behavior of hingeless rotor blades as shown in Fig. 1, with actively controlled flaps; as well as blades with advanced geometry tips as shown in Fig. 2. The key ingredients of the aeroelastic response analysis are: (1) the structural dynamic model, (2) the unsteady aerodynamic model and (3) a coupled trim/aeroelastic response procedure that is required for the computation of the 
steady state blade response. The aeroelastic response analysis and overviews of the blade stress calculations and aeroelastic stability in hover analysis are described next.

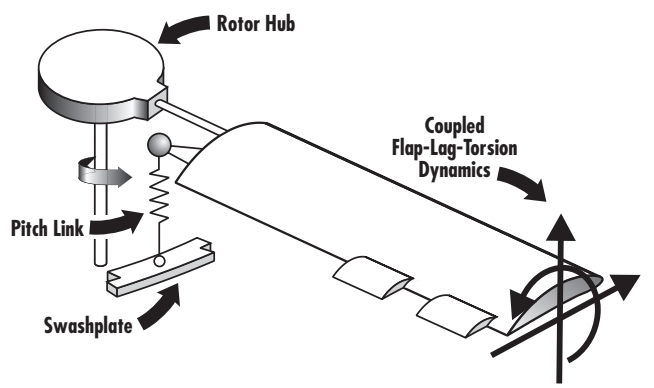

Figure 1. Helicopter rotor blade with trailing edge flaps.

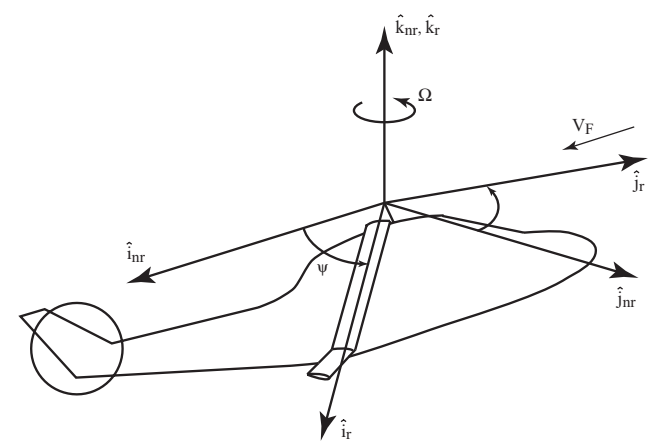

Figure 2. A blade with advanced geometry tip.

\section{II.A. Structural Dynamic Model}

The structural dynamic model is based on an analysis developed by Yuan and Friedmann ${ }^{19,25}$ which is capable of modeling composite blades with transverse shear deformations, cross-sectional warping, and swept tips. This study is limited to the behavior of isotropic blades with spanwise varying properties. The equations of motion are formulated using a finite element discretization of Hamilton's principle, with the assumption that the blade undergoes moderate deflections. The beam type finite elements used for the discretization have 23 nodal degrees of freedom. Normal modes are used to reduce the number of structural degrees of freedom. In this study, eight modes are used: the first 3 flap modes, first 2 lead-lag modes, first 2 torsional modes, and the first axial mode.

\section{II.B. Aerodynamic Model}

The attached flow blade section aerodynamics are calculated using a rational function approach (RFA). ${ }^{20,26}$ The RFA approach is a two-dimensional unsteady time-domain theory that accounts for compressibility as well as variations in the oncoming flow velocity. This two-dimensional aerodynamic model is linked to an enhanced free-wake model which provides a non-uniform inflow distribution at closely spaced azimuthal steps. ${ }^{27-29}$ Although the simulation code can also account for dynamic stall at high advance ratios, ${ }^{22}$ dynamic stall was not considered in this paper because the vibration levels being approximated are those due to blade vortex interaction (BVI), which occurs at low advance ratios.

\section{II.C. Coupled Trim/Aeroelastic Response}

The combined structural and aerodynamic equations form a system of coupled ordinary differential equations that are cast into first order state variable form ${ }^{20}$ and integrated in the time domain using the AdamsBashforth predictor-corrector algorithm. A propulsive trim procedure, where six equilibrium equations (three forces and three moments) are enforced, is used in this study. ${ }^{18,30}$ The trim equations are solved in a coupled manner with the aeroelastic equations of motion. The vibratory hub shears and moments are found by integrating the distributed inertial and aerodynamic loads over the entire blade span in the rotating frame, then transforming these loads to the hub-fixed non-rotating system, and summing the contributions from each blade. ${ }^{19}$ In the process, cancellation of various terms occurs and the primary components of the hub shears and moments have a frequency of $N_{b} / \mathrm{rev}$, which is known as the blade passage frequency.

\section{II.D. Blade Stresses}

After the blade responses are obtained from the coupled trim/aeroelastic response solution, the stresses in the blade at any spanwise location can be recovered by using strain-displacement and constitutive relations. Solving for the stresses in this manner accounts for the complicated loading a blade encounters and is consistent with the structural dynamic model. The procedure for calculating stresses is as follows: 
1. For a given azimuth angle, the displacements at any spanwise location are calculated by the aeroelastic response code.

2. The displacements are then substituted into the nonlinear strain-displacement relations, ${ }^{19}$ giving the strains at any spanwise location.

3. Stresses are calculated from the stress-strain relations.

This calculation gives the blade stresses at any spanwise location and at any azimuth angle.

\section{II.E. Aeroelastic Stability in Hover}

The process for determining the hover stability of the blade is based on the method used in Ref. 19, and is described below:

1. The non-linear static equilibrium solution of the blade is found for a given pitch setting and uniform inflow, by solving a set of nonlinear algebraic equations. Note that uniform inflow is used only in the hover stability calculation. The forward flight analysis employs a free-wake model for inflow calculation.

2. The governing system of ordinary differential equations are linearized about the static equilibrium solution by writing perturbation equations and neglecting second-order and higher terms in the perturbed quantities. The linearized equations are rewritten in first-order state variable form.

3. The real parts of the eigenvalues of the first-order state variable matrix, $\lambda_{k}=\zeta_{k}+i \omega_{k}$, determine the stability of the system. If $\zeta_{k} \leq 0$ for all $k$, the system is stable.

For this study, the linearization process from Ref. 19 is modified to account for the aerodynamic states introduced by the RFA model. ${ }^{9}$

\section{Formulation of the Blade Optimization Problem}

The formulation of the blade optimization problem in forward flight consists of several ingredients: the objective function, design variables, and constraints. The mathematical formulation of the optimization is stated as: Find the vector of design variables $\mathbf{D}$ which minimizes the objective function, i.e. $J(\mathbf{D}) \rightarrow$ min, where the objective function consists of a combination of the $N_{b} /$ rev oscillatory hub shears and moments. For a four bladed rotor, the objective function is given by

$$
J=K_{S} \sqrt{\left(F_{4 X}\right)^{2}+\left(F_{4 Y}\right)^{2}+\left(F_{4 Z}\right)^{2}}+K_{M} \sqrt{\left(M_{4 X}\right)^{2}+\left(M_{4 Y}\right)^{2}+\left(M_{4 Z}\right)^{2}}
$$

where $K_{S}$ and $K_{M}$ are appropriately selected weighting factors.

The vector of design variables $\mathbf{D}$ consists of the thicknesses $t_{1}, t_{2}, t_{3}$, and the non-structural mass $m_{n s}$ located at the shear center, which are specified at several spanwise locations and shown in Fig. 3. The three thickness design variables were defined at the $0 \%, 25 \%, 50 \%, 75 \%$, and $100 \%$ stations, while the nonstructural mass design variable was defined at the $68 \%$ and $100 \%$ blade stations, resulting in a total of 17 design variables. These two blade stations were chosen for the non-structural mass because previous studies have shown that non-structural masses are most effective for vibration reduction when they are distributed over the outboard $1 / 3$ of the blade. ${ }^{31,32}$ The cross-sectional variables were assumed to vary linearly between stations. The non-structural mass at the elastic axis inboard of the $68 \%$ station was set to zero. The design variables have side constraints to prevent them from reaching impractical values; these are stated as

$$
\mathbf{D}_{j}^{(L)} \leq \mathbf{D} \leq \mathbf{D}_{j}^{(U)}, \quad j=1,2, \ldots, N_{d v} .
$$

In addition, four types of behavior constraints, given by

$$
g_{i}(\mathbf{D}) \leq 0, \quad i=1,2, \ldots, N_{c},
$$

are placed on the design variables. The first type of behavior constraints are frequency placement constraints, which are prescribed upper and lower bounds on the fundamental flap, lag, and torsional frequencies of the blade. The frequency placement constraints on the fundamental flap frequency are written as

$$
\mathrm{g}_{\text {flap }}(\mathbf{D})=\frac{\omega_{F 1}}{\omega_{U}}-1 \leq 0
$$


and

$$
\mathrm{g}_{\text {flap }}(\mathbf{D})=1-\frac{\omega_{F 1}}{\omega_{L}} \leq 0
$$

where $\omega_{U}$ and $\omega_{L}$ are the prescribed upper and lower bounds on the fundamental flap frequency. Similar constraints are placed on the lag and torsional frequencies, i.e. $\mathrm{g}_{\text {lag }}$ and $\mathrm{g}_{\text {torsion. }}$ In addition, all blade frequencies must differ from integer multiples of the angular velocity $-1 / \mathrm{rev}, 2 / \mathrm{rev}, 3 / \mathrm{rev}, \ldots$, etc. - to avoid undesirable resonances.

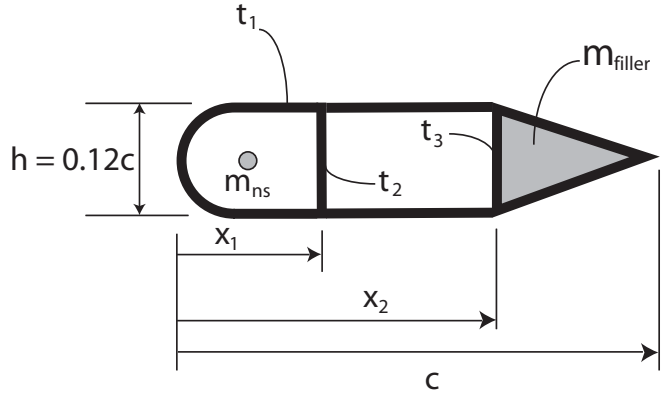

Figure 3. Simplified model of the blade structural member.

The third type of behavior constraints are aeroelastic stability margin constraints, expressed mathematically as

$$
\mathrm{g}_{k}(\mathbf{D})=\zeta_{k}+\left(\zeta_{k}\right)_{\min } \leq 0, \quad k=1,2, \ldots, N_{m}
$$

where $N_{m}$ is the number of normal modes, $\zeta_{k}$ is the real part of the hover eigenvalue for the $k^{t h}$ mode, and $\left(\zeta_{k}\right)_{\min }$ is the minimum acceptable damping level for the $k_{t h}$ mode. It should be noted that the most critical modes for stability are usually the first and second lag modes.

The final behavior constraint is a yield constraint obtained by substituting the blade stresses into Von Mises' criterion, which is expressed mathematically as

$$
\frac{2 \sigma_{x x}^{2}+6\left(\sigma_{x \eta}^{2}+\sigma_{x \zeta}^{2}\right)}{6}-\frac{\sigma_{\text {allowable }}^{2}}{3} \leq 0
$$

where $\sigma_{x x}, \sigma_{x \eta}$, and $\sigma_{x \zeta}$ are the axial and shear stresses, and $\sigma_{\text {allowable }}$ is the material yield stress divided by a factor of safety. At discrete values of the azimuth angle, Eq. (8) is evaluated at spanwise locations corresponding to the finite element nodes. The maximum evaluation of Eq. (8) is used for the constraint, and is given as

$$
\mathrm{g}(\mathbf{D})=\operatorname{MAX}\left[\frac{2 \sigma_{x x}^{2}+6\left(\sigma_{x \eta}^{2}+\sigma_{x \zeta}^{2}\right)}{6}-\frac{\sigma_{\text {allowable }}^{2}}{3}\right] \leq 0
$$

where MAX[ ] denotes the maximum value of Eq. (8) over each set of azimuth angle and blade stations at which it is evaluated. Therefore the yield constraint is enforced at the blade station and azimuth angle where the stress condition is most critical.

\section{Global Sensitivity Analysis}

Global sensitivity analysis (GSA), as presented by Sobol, ${ }^{14}$ is used to estimate the effect of different design variables on the total variability of the objective function. In this approach, the significance of a design variable is quantified by calculating the contribution of the variable to the total variance of a function. The higher the variance caused by a variable, the more significant the variable is. The advantages of conducting such an analysis include assessment of the importance of design variables and fixing nonessential variables during optimization, thus reducing the dimensionality of the problem. As proposed in this study, GSA isolates the variability in the objective function due to main effects, i.e. variability due to each design variable alone, as well as variability due to interactions between all of the design variables. A brief overview of the GSA is given below. 
A surrogate model, $p(\mathbf{x})$, of a function of a vector of independent input variables, $\mathbf{x}$ in domain $[0,1]$, is assumed and modeled as uniformly distributed random variables. The surrogate can be decomposed into the sum of functions of increasing dimensionality given by

$$
p(\mathbf{x})=p_{0}+\sum_{i=1}^{N_{d v}} p_{i}\left(x_{i}\right)+\sum_{1 \leq i<j \leq N_{d v}}^{N_{d v}} p_{i j}\left(x_{i}, x_{j}\right)+\ldots+p_{1,2, \ldots, N_{d v}}\left(x_{1}, x_{2}, \ldots, x_{N_{d v}}\right)
$$

where

$$
p_{0}=\int_{0}^{1} p(\mathbf{x}) d \mathbf{x} .
$$

By enforcing the condition $\int_{0}^{1} p_{i, \ldots, m} d x_{k}$ for $k=i, \ldots, m$, the decomposition given by Eq. (10) is unique and the total variance of $p(\mathbf{x}), V(p)$, can be decomposed in a similar fashion:

$$
V(p)=\sum_{i=1}^{N_{d v}} V_{i}+\sum_{1 \leq i<j \leq N_{d v}}^{N_{d v}} V_{i j}+\ldots+V_{1,2, \ldots, N_{d v}},
$$

where $V(p)=E\left[\left(p-p_{0}\right)^{2}\right]$, and $E[$ ] denotes the expected value operator. It can be shown that the partial variances in Eq. (12) are given by the following expressions:

$$
\begin{aligned}
V_{i} & =V\left(E\left[\left.p\right|_{x_{i}}\right]\right) \\
V_{i j} & =V\left(E\left[\left.p\right|_{x_{i}, x_{j}}\right]\right)-V_{i}-V_{j} \\
V_{i j k} & =V\left(E\left[\left.p\right|_{x_{i}, x_{j}, x_{k}}\right]\right)-V_{i j}-V_{i k}-V_{j k}-V_{i}-V_{j}-V_{k},
\end{aligned}
$$

and so on. The contribution of $x_{i}$ alone to the total variance is accounted for with $V_{i}$, while $V_{i j}, V_{i j k}$, and so on account for the variance due to interactions among $x_{i}$ and the other design variables. Note that

$$
E\left[\left.p\right|_{x_{i}}\right]=\int_{0}^{1} p_{i} d x_{i}
$$

and

$$
V\left(E\left[\left.p\right|_{x_{i}}\right]\right)=\int_{0}^{1} p_{i}^{2} d x_{i} .
$$

The total contribution of the $i^{t h}$ design variable to the total variance is given as

$$
V_{i}^{\text {total }}=V_{i}+\sum_{j, j \neq i}^{N_{d v}} V_{i j}+\sum_{j, j \neq i}^{N_{d v}} \sum_{k, k \neq i, j}^{N_{d v}} V_{i j k}+\ldots
$$

To calculate the total sensitivity of the function to any design variable, $x_{i}$, the set of design variables is divided into two subsets - the first subset contains only $x_{i}$, while the second contains all of the remaining design variables and is denoted as $B$. The total variance due to $x_{i}$ can now be rewritten as

$$
V_{i}^{\text {total }}=V_{i}+V_{i, B}
$$

where $V_{i, B}$ is the measure of the variance that is dependent on interactions between $x_{i}$ and all of the other design variables. The total variance due to the effects from all design variables is calculated from the following relation,

$$
V(p)=V_{i}+V_{B}+V_{i, B}
$$

where $V_{B}$ is the partial variance corresponding to $B$.

Finally, sensitivity indices are calculated in order to quantify the significance of a design variable. For example, the first and second order sensitivity indices are given by

$$
S_{i}=\frac{V_{i}}{V(p)}
$$


and

$$
S_{i j}=\frac{V_{i j}}{V(p)} .
$$

The first order sensitivity index accounts for the main effects of a design variable. The effects of interactions among design variables are captured by the higher order sensitivity indices. For the $i^{t h}$ design variable, the total sensitivity index is given by

$$
S_{i}^{\text {total }}=\frac{V_{i}^{\text {total }}}{V(p)} .
$$

The relative significance of each design variable can be obtained by ranking each variable according to its respective total sensitivity index, with the most significant variables corresponding to higher indices.

\section{Global Approximation Methods}

The goal in using global approximation, or surrogate, methods is to replace the "true" objective function and expensive constraints with smooth functional relationships of acceptable accuracy that can be evaluated quickly. In order to construct the surrogates, the objective function and constraints must first be evaluated over a set of design points. The surrogate is then generated by fitting the initial design points. Although function evaluations, which come from the expensive helicopter simulations, are needed to form the approximation, this initial investment of computer time is significantly less compared to global searches using non-surrogate based optimization methods. Once the surrogates have been obtained, they are used to replace the more expensive "true" objective function and constraints in the search for the optimum and in the GSA.

The surrogate objective function can be generated in two ways: (a) the vibratory hub shears and moments in Eq. (1) are replaced by surrogates and used to build the surrogate objective function, as in Eq. (22), and (b) the overall output, $J$, is approximated directly. Six responses need to be approximated in the first approach, and 1 response needs to be approximated in the second approach. Both methods will be considered in this study.

$$
\hat{J}=K_{S} \sqrt{\left(\hat{F}_{4 X}\right)^{2}+\left(\hat{F}_{4 Y}\right)^{2}+\left(\hat{F}_{4 Z}\right)^{2}}+K_{M} \sqrt{\left(\hat{M}_{4 X}\right)^{2}+\left(\hat{M}_{4 Y}\right)^{2}+\left(\hat{M}_{4 Z}\right)^{2}} .
$$

The stress constraint is the only constraint which requires a forward flight simulation, and is therefore the only computationally expensive constraint. Consequently, a surrogate constraint is used in place of Eq. (9) during optimization. Since the cost of fitting and optimizing the surrogates is generally much less than generating the initial data set, multiple surrogates are considered in order to fully utilize the sample data. Descriptions of the methods for constructing the global approximations are given below.

\section{V.A. Design of Computer Experiments}

When the initial data set is produced by a deterministic computer code (as is the case in the vibration reduction problem), the term "design of computer experiments," is more appropriate than design of experiments. ${ }^{8,34}$ The distinction is necessary because in physical experiments there is measurement error and other random sources of noise that cannot be controlled, which affects the choice of the design point. However, in computer experiments, there is no random error; i.e., for a deterministic computer code, a given input will always yield the same output. Thus, the design of computer experiments need only be space-filling. Figure 4 illustrates the difference between a conventional design of experiment and a space-filling design. In the figure, locations of design points where experiments are to be conducted, which in this case represent design points where aeroelastic response simulations are performed, are shown for a design space which has two design variables.

A commonly used space-filling design is Latin hypercube sampling (LHS). ${ }^{35}$ In LHS, each design variable is partitioned into $N_{s p}$ equally spaced sections, or strata. Every design variable $D_{i}$, where $i=1,2, \ldots, N_{d v}$, is sampled once in each strata, which forms $N_{d v}$ vectors of size $N_{s p}$. The components of the $N_{d v}$ vectors are then randomly combined to form an $N_{s p} \times N_{d v}$ matrix known as a Latin hypercube, where each row corresponds to a design point at which a computer experiment is performed. A major disadvantage of Latin hypercube sampling is that design points can cluster together due to the random process by which design points are created. To prevent this, optimal Latin hypercube $(\mathrm{OLH})^{6}$ sampling is used in this study to ensure a more uniform (or space-filling) design of computer experiment. Optimal Latin hypercube sampling creates 

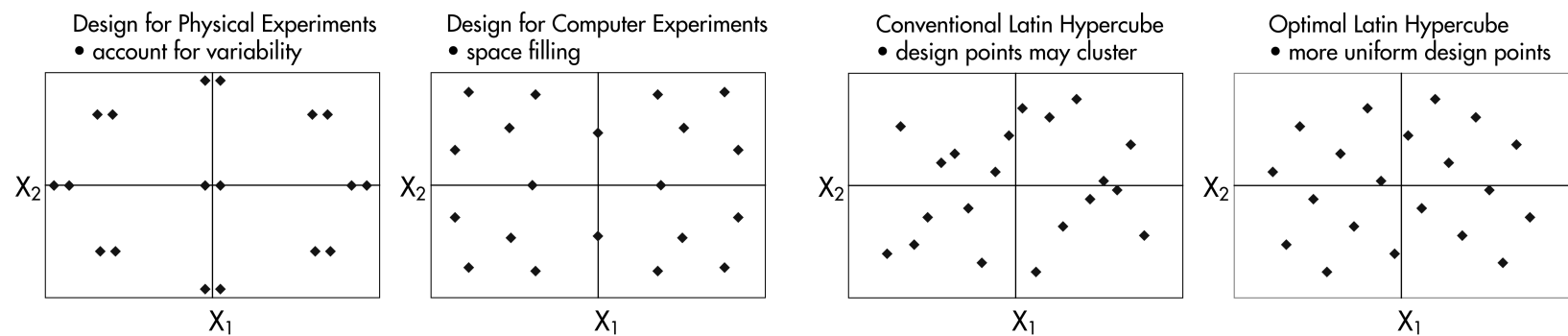

Figure 4. Design of physical experiment vs. design of computer experiment.

Figure 5. Conventional LH vs. Optimal LH in two dimensional design space.

a more uniform design than conventional LHS by maximizing a spreading criteria, rather than randomly creating design points from the samples. Figure 5 illustrates the difference between a conventional Latin hypercube and an optimal Latin hypercube. In this study, the OLH algorithm from the iSIGHT software package is used. ${ }^{36,37}$ Methods for fitting the data points in the OLH are described next.

\section{V.B. Polynomial Response Surfaces}

Suppose a deterministic function of $N_{d v}$ design variables, that needs to be approximated, has been evaluated at $N_{s p}$ sample points. Sample point $i$ is denoted $\mathbf{x}^{(i)}=\left(x_{1}^{(i)}, \ldots, x_{N_{d v}}^{(i)}\right)$ and the associated response is given by $y_{i}=y\left(\mathbf{x}^{(i)}\right)$ for $i=1, \ldots, N_{s p}$. A polynomial regression approximation to $y(\mathbf{x})$ can be written as

$$
y(\mathbf{x})=\hat{y}(\mathbf{x})+\epsilon_{\mathrm{pr}}
$$

where $\hat{y}(\mathbf{x})$ is the function chosen to approximate the true response $y(\mathbf{x})$, and $\epsilon_{\mathrm{pr}}$ is the error associated with the approximation. It is important to note that the errors are assumed to be independent; i.e. the errors at two points close together will not necessarily be close. This assumption will be revisited when considering kriging. In this study, $2^{\text {nd }}$ order polynomials are used for $\hat{y}(\mathbf{x})$. The least squares regression approximation is given $\operatorname{as}^{38}$

$$
\hat{y}_{\text {poly }}=\beta_{0}+\sum_{i=1}^{N_{d v}} \beta_{i} x_{i}+\sum_{i=1}^{N_{d v}} \sum_{j=1, i<j}^{N_{d v}} \beta_{i j} x_{i} x_{j}+\sum_{i=1}^{N_{d v}} \beta_{i i} x_{i}^{2} .
$$

In addition to Eq. (24), a reduced term polynomial surrogate in which statistically insignificant terms are removed is considered. The reduced term polynomial is obtained by sequentially removing coefficients with t-statistics less than 1 from the full term polynomial.

\section{V.C. Kriging Surrogates}

Kriging is based on the fundamental assumption that errors are correlated, which is in contrast to the assumption of independent or uncorrelated errors made in polynomial regression. This implies that one assumes the errors at two points close together will be close. In fact, the assumption that the errors are uncorrelated is only appropriate when the sources of error are random, such as in the case of measurement error or noise. In the case of deterministic computer simulations, there is no source of random error. Therefore, it is more reasonable to assume that the error terms will be correlated and that this correlation is higher the closer two points are to each other.

In kriging, the unknown function $y(\mathbf{x})$ is assumed to be of the form

$$
y(\mathbf{x})=f(\mathbf{x})+Z(\mathbf{x})
$$

where $f(\mathbf{x})$ is an assumed function (usually polynomial form) and $Z(\mathbf{x})$ is a realization of a stochastic (random) process which is assumed to be a Gaussian process with zero mean and variance of $\sigma_{v a r}^{2}$ (i.e. $Z(\mathbf{x})$ follows a normal, or Gaussian, distribution. ${ }^{39,40}$ The function $f(\mathbf{x})$ can be thought of as a global approximation of $y(\mathbf{x})$, while $Z(\mathbf{x})$ accounts for local deviations which ensure that the kriging model interpolates 
the data points exactly. The covariance matrix of $Z(\mathbf{x})$, which is a measure of how strongly correlated two points are, is given by

$$
\operatorname{Cov}\left[Z\left(\mathbf{x}^{(i)}\right), Z\left(\mathbf{x}^{(j)}\right)\right]=\sigma_{v a r}^{2} \mathbf{R}_{\mathrm{krg}}
$$

where each element of the $N_{s p} \times N_{s p}$ correlation matrix $\mathbf{R}_{\text {krg }}$ is given by

$$
\left(R_{\mathrm{krg}}\right)_{i j}=R_{\mathrm{krg}}\left(\mathbf{x}^{(i)}, \mathbf{x}^{(j)}\right)
$$

and $R_{\mathrm{krg}}\left(\mathbf{x}_{i}, \mathbf{x}_{j}\right)$ is a correlation function which accounts for the effect of each interpolation point on every other interpolation point. This function is called the spatial correlation function (SCF) and is chosen by the user. The most commonly used SCF is the Gaussian correlation function,

$$
R_{\mathrm{krg}}\left(\mathbf{x}^{(i)}, \mathbf{x}^{(j)}\right)=\exp \left[-\sum_{k=1}^{N_{d v}} \vartheta_{k}\left|x_{k}{ }^{(i)}-x_{k}{ }^{(j)}\right|^{p_{k}}\right],
$$

which is also employed in this study. The Gaussian SCF is dependent on the distance between two points. As two points move closer to each other, $\left|x_{k}{ }^{(i)}-x_{k}{ }^{(j)}\right| \rightarrow 0$, and Eq. (28) approaches unity which is the maximum value of the Gaussian SCF. In other words, the Gaussian SCF recovers the intuitive property that the closer two points are to each other, the greater the correlation between the points.

The fitting parameters $\vartheta_{k}$ and $p_{k}$ are unknown correlation parameters which need to be determined. In order to determine these parameters, the form of $f(\mathbf{x})$ needs to chosen. In this study, $f(\mathbf{x})$ is assumed to be a linear polynomial and is given by

$$
f(\mathbf{x})=f_{x}^{\mathrm{T}} \beta
$$

where $f_{x}^{\mathrm{T}}$ is a $1 \times N_{\text {basis }}$ vector, $N_{\text {basis }}$ is the number of basis functions associated with the linear polynomial, and $\beta$ is a $N_{\text {basis }} \times 1$ vector of coefficients. Similarly, $\mathbf{F}(\mathbf{x})$ can be defined as an $N_{s p} \times N_{\text {basis }}$ matrix where the $i^{\text {th }}$ row corresponds to the evaluation of the $N_{\text {basis }}$ functions at the $i^{\text {th }}$ sample point. Furthermore, a common simplification is to fix all $p_{k}=2$. In Ref. 9, allowing $p_{k}$ to vary offered no clear benefit over fixing $p_{k}=2$. Therefore, fixing all $p_{k}=2$ is employed in this study. In order to find $\vartheta_{k}$, the generalized least square estimates of $\beta$ and $\sigma_{v a r}^{2}$, denoted by $\hat{\beta}$ and $\hat{\sigma}_{v a r}^{2}$ respectively, are employed: ${ }^{39,40}$

$$
\hat{\beta}=\left(\mathbf{F}^{\mathrm{T}}\left(\mathbf{R}_{\mathrm{krg}}\right)^{-1} \mathbf{F}\right)^{-1} \mathbf{F}^{\mathrm{T}}\left(\mathbf{R}_{\mathrm{krg}}\right)^{-1} \mathbf{y}
$$

and

$$
\hat{\sigma}_{v a r}^{2}=\frac{(\mathbf{y}-\mathbf{F} \hat{\beta})^{\mathrm{T}}\left(\mathbf{R}_{\mathrm{krg}}\right)^{-1}(\mathbf{y}-\mathbf{F} \hat{\beta})}{N_{s p}}
$$

where $\mathbf{y}$ is a vector of observed function outputs at the interpolation points; both vectors are of length $N_{s p}$. With $\hat{\sigma}_{v a r}^{2}$ and $\hat{\beta}$ known, $\vartheta_{k}$ are found such that a likelihood function ${ }^{39,40}$ is maximized. The likelihood function, given in Eq. (32), is a measure of the probability of the sample data being drawn from a probability density function associated with a Gaussian process. Since the stochastic process associated with kriging has been assumed to be a Gaussian process, one seeks the set of $\vartheta_{k}$ that maximize the probability that the interpolation points have been drawn from a Gaussian process.

$$
-\frac{\left[N_{s p} \ln \left(\hat{\sigma}_{v a r}^{2}\right)+\ln \left|\mathbf{R}_{\mathrm{krg}}\right|\right]}{2}
$$

The maximum likelihood estimates (MLE's) of $\vartheta_{k}$ represent the "best guesses" of the fitting parameters. Any values of $\vartheta_{k}$ would result in a surrogate which interpolates the sample points exactly, but the "best" kriging surrogate is found by optimizing the likelihood function. This auxiliary optimization process can result in significant fitting time depending on the size of the system. Due to the optimization process needed to create the kriging surrogate, kriging is only appropriate when the time needed to generate the interpolation points is much larger than the time to interpolate the data - which is the case in the helicopter vibration problem. With all parameters known, the kriging approximation to a function $y(\mathbf{x})$ can be written as $^{34,39-41}$

$$
\hat{y}_{\mathrm{krg}}=f_{x}^{\mathrm{T}} \hat{\beta}+\mathbf{r}_{\mathrm{krg}}(\mathbf{x})^{\mathrm{T}}\left(\mathbf{R}_{\mathrm{krg}}\right)^{-1}(\mathbf{y}-\mathbf{F} \hat{\beta})
$$

where

$$
\mathbf{r}_{\mathrm{krg}}(\mathbf{x})=\left[R_{\mathrm{krg}}\left(\mathbf{x}, \mathbf{x}^{(1)}\right), R_{\mathrm{krg}}\left(\mathbf{x}, \mathbf{x}^{(2)}\right), \ldots, R_{\mathrm{krg}}\left(\mathbf{x}, \mathbf{x}^{\left(N_{s p}\right)}\right)\right]^{\mathrm{T}}
$$

The column vector $\mathbf{r}_{\mathrm{krg}}(\mathbf{x})$ of length $N_{s p}$ is the correlation vector between an arbitrary point $\mathbf{x}$ and the interpolation points, $\mathbf{x}^{(1)}, \ldots, \mathbf{x}^{\left(N_{s p}\right)}$. 


\section{V.D. Radial Basis Neural Networks}

Radial basis neural networks (RBNN) approximate a function as a weighted sum of radial basis functions, also known as neurons.

$$
\hat{y}_{\mathrm{RBNN}}=\sum_{i=1}^{N_{R B F}} \alpha_{i} \phi_{\mathrm{RBNN}}(\mathbf{x})
$$

where $\phi_{\mathrm{RBNN}}(\mathbf{x})$ is the response of the radial basis function at $\mathbf{x}$, and $\alpha_{i}$ is the weight associated with the radial basis function. In this study, the MATLAB routine newrb is used to construct the RBNN. Gaussian function's given by Eq. (36) are used for the neurons.

$$
\phi_{\mathrm{RBNN}}(\eta)=\exp \left(-\eta^{2}\right)
$$

In this case, the dummy variable $\eta$ would be $\left(\tau\left\|\mathbf{x}-\mathbf{x}^{(i)}\right\|\right)$, where $\left\|\mathbf{x}-\mathbf{x}^{(i)}\right\|$ is the Euclidean distance between two vectors. The parameter $\tau$ is inversely related to the user defined parameter denoted spread, which controls the radius of influence for each neuron. Specifically, the radius of influence is the distance at which the output of a neuron reaches a certain small value corresponding to half of the spread parameter. A high spread would cause the neuron responses to be smooth, and a low spread would result in highly non-linear responses. The spread is set to 0.5 in this study. The number of radial basis functions and associated weights are determined by satisfying the user defined error goal for the mean square error in the approximation. The goal parameter is set to the square of $5 \%$ of the mean response in this study.

\section{V.E. Weighted Average Surrogates}

In addition to the individual surrogates described above, a weighted average surrogate based on the implementation described in Ref. 10 will be employed. The weighted average surrogate is formulated as a weighted sum of the three individual approximation methods, i.e.

$$
\hat{y}_{\mathrm{wTA}}=w_{\mathrm{poly}} \hat{y}_{\mathrm{poly}}+w_{\mathrm{krg}} \hat{y}_{\mathrm{krg}}+w_{\mathrm{RBNN}} \hat{y}_{\mathrm{RBNN}}
$$

where $w_{\text {poly }}, w_{\text {krg }}$, and $w_{\text {RBNN }}$ are the weights associated with each surrogate. The weights are calculated in such a way that they (a) reflect the confidence in each individual surrogate and (b) filter out adverse effects associated with individual surrogates which represent the sample data well, but predict poorly at designs not included in the sample data. Furthermore, the sum of the weights in Eq. (37) are constrained to sum to 1 so that if all of the individual surrogates give the same output at some input, then the weighted surrogate will also recover this output. A weight scheme which satisfies these issues is given below. ${ }^{10}$

$$
w_{i}=\frac{w_{i}^{*}}{\sum_{i}^{N_{s m}} w_{i}^{*}}
$$

where

$$
\begin{gathered}
w_{i}^{*}=\left(E_{i}+d_{1} E_{\text {avg }}\right)^{d_{2}}, \quad d_{1}<1, d_{2}<0 \\
E_{\text {avg }}=\sum_{i}^{N_{s m}} E_{i} / N_{s m}
\end{gathered}
$$

and $N_{s m}$ is the number of surrogate models. The weights are based on a global data based error measure for each surrogate, denoted $E_{i}$. In this study, the generalized mean square error(GMSE) based on leave-one-out cross-validation is used as the error measure, and thus

$$
E_{i}=\sqrt{G M S E_{i}} .
$$

Details on how the GMSE is determined are given in the Appendix. In Eq. (39), $d_{1}$ and $d_{2}$ are user defined parameters which control the relative influence of the individual surrogate error, $E_{i}$, and the average of the individual errors, $E_{\text {avg }}$, on the weight. Small values of $d_{1}$ and large negative values of $d_{2}$ result in high weights for the best individual surrogate, which satisfies the first goal mentioned above for determining the weights. Large values of $d_{1}$ and small negative values of $d_{2}$ result in more emphasis on the average of the error, which would protect against surrogates which may predict well at sample data points, but give poor predictions at unsampled locations in the design space. Based on a parametric study conducted in Ref. 10, $d_{1}$ and $d_{2}$ are set to 0.05 and -1 respectively. Note that the intuitive property that the higher the error, the lower the weight corresponding to a surrogate is recovered since $d_{2}<0$ 


\section{Results}

This section presents accuracy measures of the approximation methods that have been described, vibration reduction results using surrogate objective functions, and GSA results. The helicopter configuration used in all computations is given in Table 1. The simulations are conducted at an advance ratio of 0.15 and descent angle of $6.5^{\circ}$, where high vibration levels due to strong blade vortex interaction (BVI) are encountered. Figure 6 illustrates a helicopter in descent; this figure is also employed for the propulsive trim calculation.

\begin{tabular}{|c|c|}
\hline \multicolumn{2}{|l|}{ Dimensional Data } \\
\hline$R=4.91 \mathrm{~m}$ & $\Omega=425 \mathrm{RPM}$ \\
\hline Non-Dimensional Data & \\
\hline$\overline{N_{b}=4}$ & $c=0.05498 R$ \\
\hline$\beta_{p}=0.0^{\circ}$ & $C_{d o}=0.01$ \\
\hline$\theta_{\mathrm{pt}}=0^{\circ}$ & $\alpha_{\mathrm{d}}=6.5^{\circ}$ \\
\hline$\mu=0.15$ & $C_{W}=0.005$ \\
\hline$\sigma=0.07$ & $C_{d f}=0.01$ \\
\hline$X_{F A}=0.0$ & $Z_{F A}=0.3$ \\
\hline$X_{F C}=0.0$ & $Z_{F C}=0.3$ \\
\hline
\end{tabular}

Table 1. Rotor and helicopter parameters needed for the computations.

\begin{tabular}{|c|c|}
\hline \multicolumn{2}{|c|}{ Aluminum Material Properties } \\
\hline \multicolumn{2}{|l|}{$E=70.7 \mathrm{GPa}$} \\
\hline \multicolumn{2}{|l|}{$\nu=0.33$} \\
\hline \multicolumn{2}{|c|}{$\rho_{\text {struct }}=2700 \mathrm{~kg} / \mathrm{m}^{3}$} \\
\hline \multicolumn{2}{|c|}{$\sigma_{Y}=324 \mathrm{MPa}$} \\
\hline \multicolumn{2}{|c|}{ Non-structural Filler Mass Density } \\
\hline \multicolumn{2}{|c|}{$\rho_{\text {filler }}=237.4 \mathrm{~kg} / \mathrm{m}^{3}$} \\
\hline \multicolumn{2}{|c|}{ Locations of the Vertical Walls } \\
\hline$x_{1}=65.4 \mathrm{~mm}$ & $x_{2}=111.6 \mathrm{~mm}$ \\
\hline
\end{tabular}

Table 2. Fixed parameters defining the structure and cross section.

In addition to the information provided in Table 1, additional information is needed for the fixed cross sectional parameters, objective function, constraints, and the finite element discretization of the blade. The material properties and the chordwise locations of the vertical walls are given in Table 2. The weighting factors in the objective function, $K_{S}$ and $K_{M}$, are selected to be 1 . These weighting factors result in an objective function which represents the sum of the 4/rev oscillatory hub shear resultant and the $4 / \mathrm{rev}$ oscillatory hub moment resultant in the hub-fixed non-rotating frame. For this study, the following side constraints are enforced:

$$
\begin{gathered}
1.0 \mathrm{~mm} \leq t_{1} \leq 8.0 \mathrm{~mm} \\
1.0 \mathrm{~mm} \leq t_{2}, t_{3} \leq 12.0 \mathrm{~mm} \\
0.0 \leq m_{n s} / m_{0} \leq 0.25
\end{gathered}
$$

The upper and lower bounds used for the frequency placement constraints, /rev, are given below.

$$
\begin{aligned}
& 0.60 \leq \omega_{L 1} \leq 0.80 \\
& 1.05 \leq \omega_{F 1} \leq 1.20 \\
& 2.50 \leq \omega_{T 1} \leq 6.50
\end{aligned}
$$

In the aeroelastic stability constraints given by Eq. (7), the minimum acceptable damping for all modes, $\left(\zeta_{k}\right)_{\min }$, is chosen to be 0.01 . Additionally, the constraints are modified for the $2^{\text {nd }}$ lag mode, which can sometimes be slightly unstable. To prevent this situation, a small amount of structural damping is added to this mode. For this study, $0.5 \%$ structural damping is added to stabilize the $2^{\text {nd }}$ lag mode of the baseline blade. For the stress constraint, a factor of safety of 1.5 is used. The rotor blade was discretized into the 6 finite elements shown in Fig. 7.

In this study, two sets of fitting points are used to build the surrogates - a 300 point optimal Latin hypercube (OLH) and a 500 point OLH. From the 300 point OLH, 283 points had converged trim solutions and were used to build the surrogates; while out of the 500 point OLH, 484 points had converged trim solutions. 


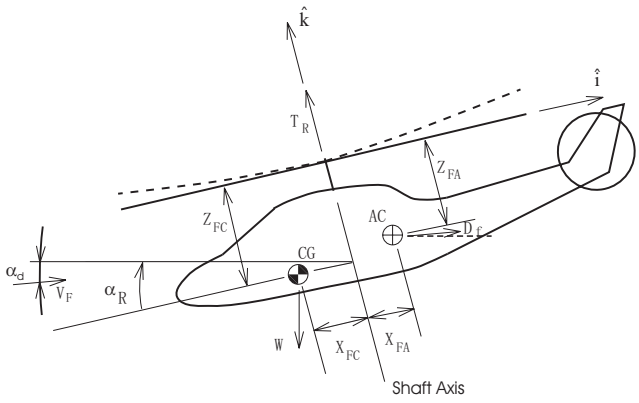

Figure 6. Helicopter in descent flight condition.

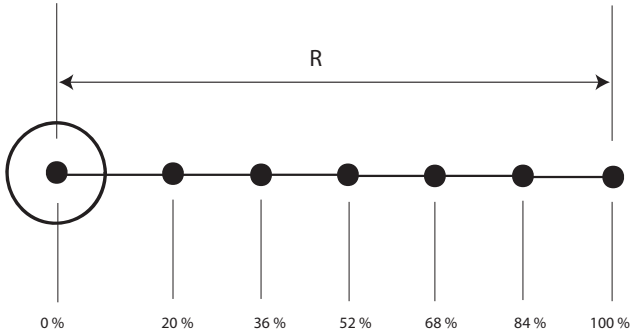

Figure 7. Finite element node locations.

\section{VI.A. Weighted Average Surrogate Construction}

The weight coefficients necessary to define the weighted average surrogates are given in Tables $3-4$. The weight coefficients obtained when using the full term polynomial response surface are given in Table 3. Generally, the kriging surrogate has the highest weight for all responses and both sample sizes in Table 3. When the reduced term polynomial is used in place of the full term polynomial, as shown in Table 4, the polynomial is weighted the most for all responses and sample sizes. Furthermore, Tables $3-4$ show that the radial basis neural network generally has the lowest weight.

Table 3. Weight coefficients of the weighted average surrogates, with full term polynomial response surfaces

\begin{tabular}{cccccccccc}
\hline $\begin{array}{c}\text { Weight } \\
\text { Coefficient }\end{array}$ & $\begin{array}{c}\text { Sample } \\
\text { Size }\end{array}$ & $\mathbf{F}_{\mathbf{4 X}}$ & $\mathbf{F}_{\mathbf{4 Y}}$ & $\mathbf{F}_{\mathbf{4 Z}}$ & $\mathbf{M}_{\mathbf{4 X}}$ & $\mathbf{M}_{\mathbf{4 Y}}$ & $\mathbf{M}_{\mathbf{4 Z}}$ & $\mathbf{J}$ & $\begin{array}{c}\text { Yield } \\
\text { Constraint }\end{array}$ \\
\hline$w_{\text {poly }}$ & 283 & 0.407 & 0.395 & 0.322 & 0.374 & 0.306 & 0.291 & 0.333 & 0.353 \\
$w_{k r g}$ & 283 & 0.478 & 0.473 & 0.458 & 0.460 & 0.449 & 0.412 & 0.461 & 0.443 \\
$w_{R B N N}$ & 283 & 0.115 & 0.132 & 0.219 & 0.167 & 0.245 & 0.297 & 0.206 & 0.205 \\
\hline$w_{p o l y}$ & 484 & 0.422 & 0.436 & 0.360 & 0.378 & 0.340 & 0.333 & 0.379 & 0.400 \\
$w_{k r g}$ & 484 & 0.449 & 0.422 & 0.425 & 0.448 & 0.405 & 0.381 & 0.419 & 0.424 \\
$w_{R B N N}$ & 484 & 0.129 & 0.142 & 0.215 & 0.175 & 0.255 & 0.286 & 0.203 & 0.176 \\
\hline \hline
\end{tabular}

Table 4. Weight coefficients of the weighted average surrogates, with reduced term polynomial response surfaces

\begin{tabular}{cccccccccc}
\hline $\begin{array}{c}\text { Weight } \\
\text { Coefficient }\end{array}$ & $\begin{array}{c}\text { Sample } \\
\text { Size }\end{array}$ & $\mathbf{F}_{\mathbf{4 X}}$ & $\mathbf{F}_{\mathbf{4 Y}}$ & $\mathbf{F}_{\mathbf{4 Z}}$ & $\mathbf{M}_{\mathbf{4 X}}$ & $\mathbf{M}_{\mathbf{4 Y}}$ & $\mathbf{M}_{\mathbf{4 Z}}$ & $\mathbf{J}$ & $\begin{array}{c}\text { Yield } \\
\text { Constraint }\end{array}$ \\
\hline$w_{\text {poly }}$ & 283 & 0.497 & 0.493 & 0.431 & 0.472 & 0.406 & 0.401 & 0.443 & 0.433 \\
$w_{k r g}$ & 283 & 0.406 & 0.396 & 0.385 & 0.388 & 0.385 & 0.349 & 0.385 & 0.388 \\
$w_{R B N N}$ & 283 & 0.097 & 0.110 & 0.183 & 0.140 & 0.209 & 0.250 & 0.172 & 0.179 \\
\hline$w_{p o l y}$ & 484 & 0.465 & 0.474 & 0.409 & 0.427 & 0.384 & 0.377 & 0.424 & 0.436 \\
$w_{k r g}$ & 484 & 0.415 & 0.394 & 0.393 & 0.413 & 0.378 & 0.356 & 0.388 & 0.399 \\
$w_{R B N N}$ & 484 & 0.120 & 0.132 & 0.199 & 0.161 & 0.238 & 0.267 & 0.188 & 0.165 \\
\hline \hline
\end{tabular}

\section{VI.B. Surrogate Accuracy Results}

The predictive capabilities of the individual and weighted average surrogates were quantified using a set of data points not included in the construction of the surrogates. The predicted responses from the surrogates were then compared to the "actual" responses at the test points. The test points came from a 200 point OLH, of which 197 had converged trim solutions. None of the blade designs from the 197 test points were 
coincident with the blade designs from the two OLH's used to create the surrogates. Using the test points, the absolute percent error is given by

$$
\varepsilon_{i}=\frac{\left|y^{(i)}-\hat{y}^{(i)}\right|}{\bar{y}}
$$

where $y^{(i)}$ is the "actual" response computed by the helicopter simulation, $\hat{y}^{(i)}$ is the response predicted by the surrogate at the $i^{\text {th }}$ test point, For the vibratory load errors, $\bar{y}$ is the mean of the absolute values of the responses from the 197 test points, while for the errors in the surrogate stress constraint,

$$
\bar{y}=\frac{\sigma_{\text {allowable }}^{2}}{3} .
$$

Based on Eq. (48), the average and maximum percent errors are:

$$
\begin{gathered}
\varepsilon_{a v g}=\frac{\sum_{i=1}^{N_{t p}} \varepsilon_{i}}{N_{t p}} \\
\varepsilon_{\text {max }}=\operatorname{Max}\left\{\varepsilon_{1}, \ldots, \varepsilon_{N_{t p}}\right\}
\end{gathered}
$$

where $N_{t p}$ is the number of test points. The maximum percent error represents the worst prediction error, while the average percent error represents the surrogate's predictive capability over the entire design space since all 197 test points are included.

The average and maximum errors are given in Figures 8 and 9 respectively for 7 responses -6 underlying hub shears and moments and the stress constraint. Note that "(red)" indicates the use of reduced term polynomial surrogates. Among the individual surrogates for each response, the lowest average errors range from $2-53 \%$ and the highest average errors range from $5-69 \%$ for the 283 sample set. By comparison, the average errors of the weighted average surrogates range from 2-53\%. These results indicate that the weighted average surrogates performed as well as the best individual surrogates, while protecting against the poor performance of the worst approximation method for all 7 responses. A similar result is obtained for the 484 point sample set where the lowest average errors in Figure 8(b) range from 3-55\% while the average errors of the weighted average surrogates range from $2-52 \%$.

For the 283 point sample set, the kriging surrogate has the lowest average error of the individual surrogates for each response, while for some responses with the 484 point sample set, the polynomials corresponded to the lowest average errors. So the choice of the "best" surrogate in terms of approximating over the entire design space is dependent on the sample size for the responses considered in this study. However, the weighted average surrogates performed as well as the best approximation methods regardless of sample size, which exemplifies the advantage of this approach.

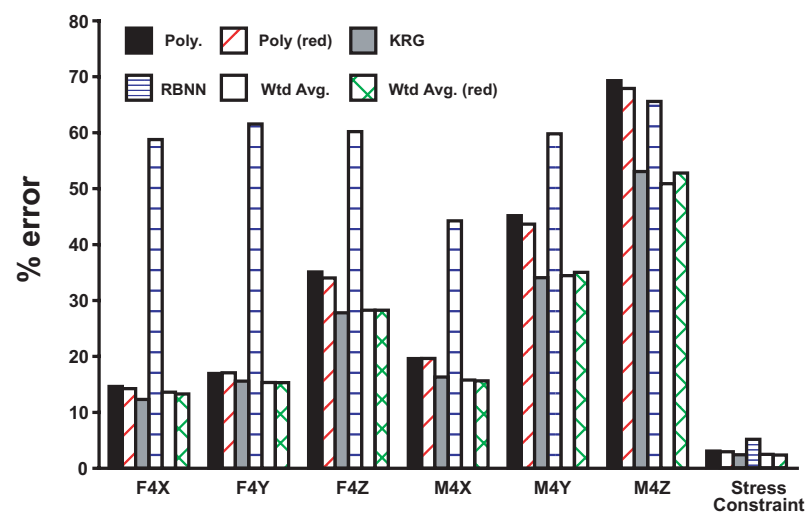

(a) 283 Sample Points

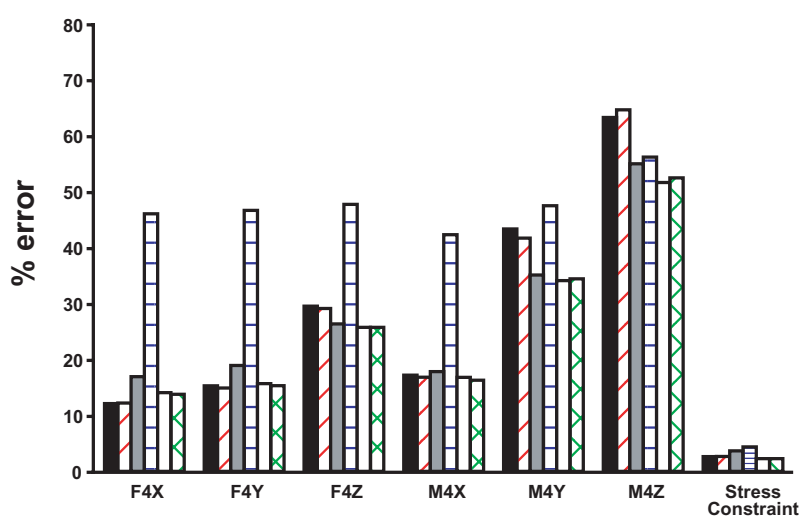

(b) 484 Sample Points

Figure 8. Average errors of the underlying vibratory loads and stress constraint.

Similar to the results for average error, Figure 9 shows that the weighted average surrogates typically perform as well as the best individual surrogate in terms of maximum error. For the 283 and 484 point sample sets, the lowest maximum errors are $32-331 \%$ and $30-313 \%$ respectively, while the weighted average surrogate maximum errors range from $37-368 \%$ and $40-339 \%$. The largest difference between the best 
individual surrogates and the weighted average models occurs when approximating the $M_{4 Y}$ and $M_{4 Z}$ responses. However, even for these two responses, the weighted average surrogates still protected against the worst individual model, which are the radial basis neural networks. It is worth noting that even though the weighted average surrogates are constructed using a global error measure - see Eq. (41) - they still perform well compared to the individual surrogate models in terms of maximum error, which is a local error measure.

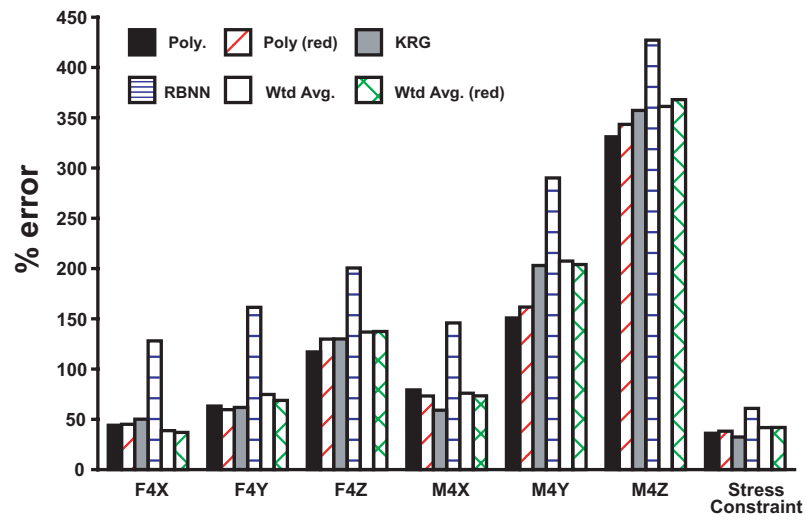

(a) 283 Sample Points

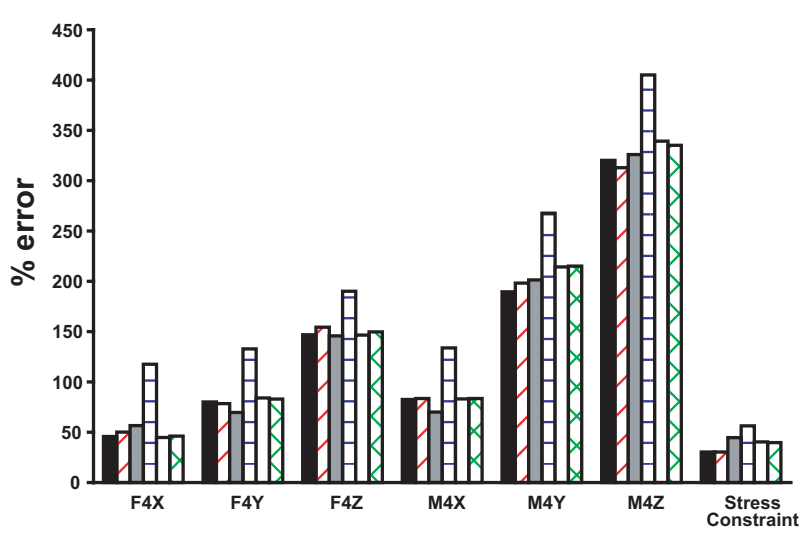

(b) 484 Sample Points

Figure 9. Maximum errors of the underlying vibratory loads and stress constraint.

The errors in the surrogate objective function are given in Figures 10 and 11. The surrogate objective function was generated with two approaches: (a) combining the underlying surrogate hub shears and moments to form the approximate objective function as in Eq. (22) and (b) by directly fitting the outputs for $J$ at the sample points. In terms of average error, the kriging surrogate is the best individual fitting method, with 19-24\% errors for both sample sizes. For both methods of fitting the objective function and both sample sizes, the weighted average surrogates perform as well as the kriging surrogates in terms of average error. The largest difference in average error between the weighted average surrogates and the kriging surrogate is only $2 \%$ and occurs in the case of the 484 point data set when combining the underlying responses to form the approximate objective function. In terms of maximum error, the weighted surrogates never produce an error more than $24 \%$ above the error from the best individual surrogate, and always perform better than the worst surrogate. So regardless of the method used to form the approximate objective function, the weighted surrogates performed as well as the best individual model in terms of average error, and performed better than the worst surrogate in terms of maximum error.

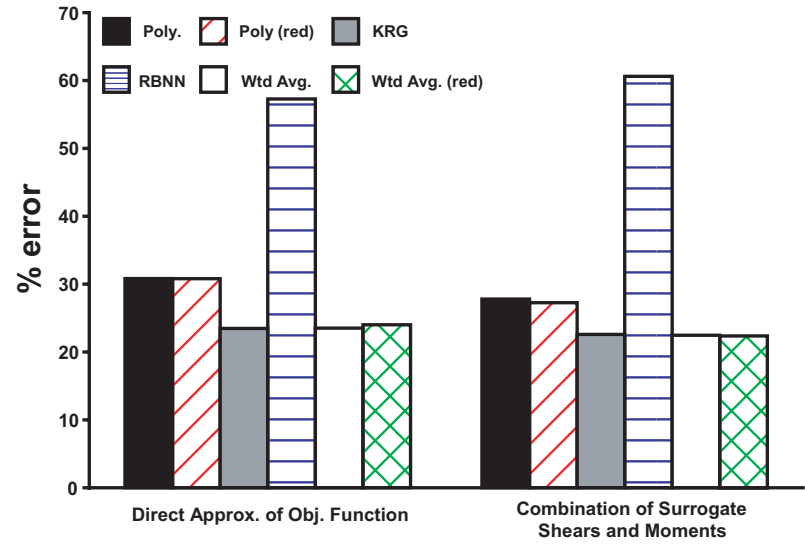

(a) 283 Sample Points

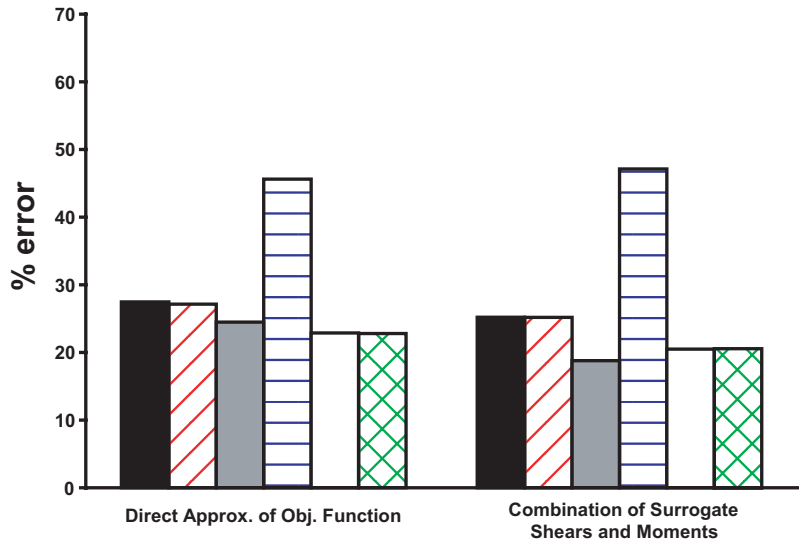

(b) 484 Sample Points

Figure 10. Average errors in the surrogate objective function.

A comparison of the errors in Figures 8 - 11 with the weight coefficients in Tables $3-4$ shows that the surrogate with the highest weight is not necessarily the most accurate when using test points to measure error. For example, consider the case of approximating the overall objective function directly. The results in 


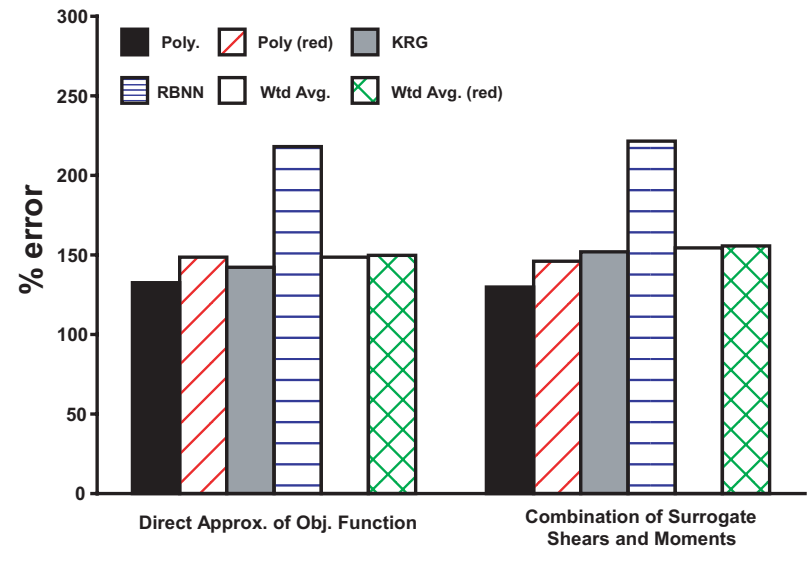

(a) 283 Sample Points

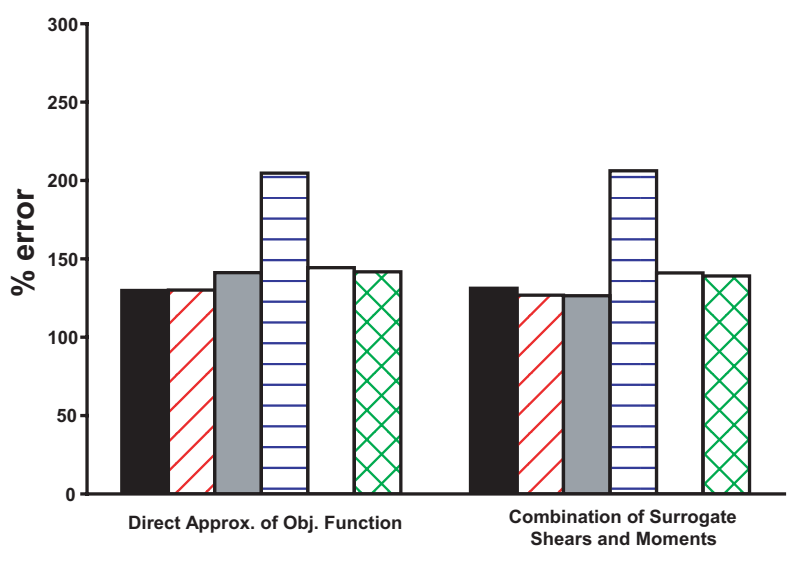

(b) 484 Sample Points

Figure 11. Maximum errors in the surrogate objective function.

Table 4 indicate that reduced term polynomials are more accurate than kriging surrogates when using the GMSE - see Appendix - as the global error measure. However, Figure 10 shows that the kriging surrogates are more accurate than the reduced term polynomials when using the average error based on test points - see Eq. 48 - to quantify error. This example illustrates a major issue with attempting to identify the most accurate individual surrogate for a given application: the most accurate approximation method may be dependent on the metric used to quantify error.

It is interesting to note that increasing the sample size from 283 to 484 generally had little effect on the accuracy of the surrogates. This indicates that for the 17 dimensional design space, increasing the number of fitting points from 283 to 484 was not sufficient to significantly enhance the surrogates' predictions of the responses considered in this study.

\section{VI.C. Optimization Results}

Optimization results based on surrogate objective functions and constraints are presented in this section. Optimization of the surrogate objective functions was conducted with the Multi-Island Genetic Algorithm in iSIGHT.

Table 5 gives the optimization results when using the underlying hub shears and moments to build the surrogate objective function. Note that vibration reduction is computed relative to the vibration levels of a baseline blade resembling an MBB BO-105 blade. The stress margin is given by

$$
1-\frac{\sqrt{\sigma_{x x}^{2}+3\left(\sigma_{x \eta}^{2}+\sigma_{x \zeta}^{2}\right)}}{\sigma_{\text {allowable }}} .
$$

A stress margin $<0$ would correspond to a design which violates the stress constraint. Table 5 shows that the best individual surrogate differs with the sample size. For the 283 point sample set the reduced term polynomial produces the best design, with $67.3 \%$ vibration reduction; while for 484 points, kriging produces the best design, with $68.7 \%$ reduction. Significant vibration reduction is also obtained with the weighted average surrogates, which produce up to $63.9 \%$ vibration reduction with 283 sample points and up to $70.2 \%$ reduction with 484 sample points. For both sample sizes, the weighted average surrogates led to better designs than the worst individual surrogate, and even led to a better design than any individual surrogate in the case of 484 sample points using the reduced term polynomial.

Optimization results corresponding to direct approximation of the overall objective function are given in Table 6. The full term polynomial led to the best design among the individual surrogates for the 283 sample set, while the radial basis neural network, which is the least accurate surrogate, led to the best design for the 484 point sample set. However, the full term polynomial is the worst individual surrogate with 484 sample points. These results represent extreme examples in the sense that the best individual surrogate for one sample set is the worst surrogate for another sample set, and the least accurate surrogate led to the best design in one instance. Even under these circumstances, the weighted average surrogates always led to 
Table 5. Comparison of predicted and "actual" vibration reductions using approximate underlying responses, along with actual stress margins.

\begin{tabular}{cccccccc}
\hline \hline Surrogate & $\begin{array}{c}\text { Sample } \\
\text { Size }\end{array}$ & $\begin{array}{c}\text { Predicted } \\
\text { Reduction }\end{array}$ & $\begin{array}{c}\text { Actual } \\
\text { Reduction }\end{array}$ & $\begin{array}{c}\text { Actual } \\
\text { Stress Margin }\end{array}$ & $\boldsymbol{\omega}_{\boldsymbol{L} 1}$ & $\boldsymbol{\omega}_{\boldsymbol{F 1} 1}$ & $\boldsymbol{\omega}_{\boldsymbol{T} \mathbf{1}}$ \\
& 283 & $100.0 \%$ & $66.4 \%$ & 0.017 & 0.671 & 1.062 & 5.036 \\
Poly. & 283 & $100.0 \%$ & $67.3 \%$ & 0.014 & 0.641 & 1.059 & 4.679 \\
Poly(red) & 283 & $94.2 \%$ & $59.0 \%$ & 0.008 & 0.613 & 1.059 & 4.176 \\
KRG & 283 & $94.9 \%$ & $53.7 \%$ & 0.016 & 0.624 & 1.059 & 4.334 \\
RBNN & 283 & $95.4 \%$ & $61.3 \%$ & 0.003 & 0.601 & 1.055 & 4.285 \\
Wtd. Avg. & 283 & $96.7 \%$ & $63.9 \%$ & 0.008 & 0.604 & 1.057 & 4.120 \\
Wtd. Avg.(red) & 484 & $100.0 \%$ & $58.9 \%$ & 0.006 & 0.608 & 1.056 & 3.958 \\
\hline Poly. & 484 & $100.0 \%$ & $62.2 \%$ & 0.007 & 0.603 & 1.056 & 3.919 \\
Poly(red) & 484 & $87.0 \%$ & $68.7 \%$ & 0.008 & 0.621 & 1.058 & 4.560 \\
KRG & 484 & $98.7 \%$ & $52.4 \%$ & 0.006 & 0.603 & 1.056 & 4.018 \\
RBNN & 484 & $88.3 \%$ & $68.1 \%$ & 0.003 & 0.618 & 1.059 & 3.866 \\
Wtd. Avg. & 484 & $89.5 \%$ & $70.2 \%$ & 0.003 & 0.615 & 1.059 & 3.796 \\
Wtd. Avg.(red) & & & & & & & \\
\hline \hline
\end{tabular}

better designs than the worst individual surrogate, and the weighted average surrogates based on full term polynomials led to better designs than any of the individual approximation methods.

Table 6. Comparison of predicted and "actual" vibration reductions when directly approximating the objective function, along with actual stress margins.

\begin{tabular}{cccccccc}
\hline \hline Surrogate & $\begin{array}{c}\text { Sample } \\
\text { Size }\end{array}$ & $\begin{array}{c}\text { Predicted } \\
\text { Reduction }\end{array}$ & $\begin{array}{c}\text { Actual } \\
\text { Reduction }\end{array}$ & $\begin{array}{c}\text { Actual } \\
\text { Stress Margin }\end{array}$ & $\boldsymbol{\omega}_{\boldsymbol{L} \mathbf{1}}$ & $\boldsymbol{\omega}_{\boldsymbol{F} \mathbf{1}}$ & $\boldsymbol{\omega}_{\boldsymbol{T} \mathbf{1}}$ \\
\hline Poly. & 283 & $394.4 \%$ & $64.4 \%$ & 0.005 & 0.610 & 1.058 & 4.330 \\
Poly(red) & 283 & $512.3 \%$ & $60.1 \%$ & 0.005 & 0.605 & 1.057 & 4.231 \\
KRG & 283 & $120.0 \%$ & $54.1 \%$ & 0.006 & 0.600 & 1.055 & 4.252 \\
RBNN & 283 & $93.9 \%$ & $57.4 \%$ & 0.009 & 0.602 & 1.055 & 4.420 \\
Wtd. Avg. & 283 & $234.9 \%$ & $70.5 \%$ & 0.008 & 0.604 & 1.055 & 4.538 \\
Wtd. Avg.(red) & 283 & $221.7 \%$ & $65.0 \%$ & 0.000 & 0.604 & 1.059 & 3.871 \\
\hline Poly. & 484 & $222.4 \%$ & $45.0 \%$ & 0.001 & 0.627 & 1.060 & 3.960 \\
Poly(red) & 484 & $207.1 \%$ & $50.0 \%$ & 0.000 & 0.600 & 1.058 & 3.710 \\
KRG & 484 & $145.1 \%$ & $55.8 \%$ & 0.000 & 0.606 & 1.057 & 3.981 \\
RBNN & 484 & $97.4 \%$ & $67.5 \%$ & 0.000 & 0.631 & 1.057 & 4.670 \\
Wtd. Avg. & 484 & $116.7 \%$ & $67.6 \%$ & 0.010 & 0.620 & 1.057 & 4.602 \\
Wtd. Avg.(red) & 484 & $129.2 \%$ & $58.8 \%$ & 0.008 & 0.625 & 1.056 & 4.380 \\
\hline \hline
\end{tabular}

Tables 5 and 6 also show that among the individual surrogates, the approximation method which led to the best design depended on the sample size. Therefore, if the best individual approximation method with one sample set were the only method used with the other sample set, inferior designs would be obtained compared to those found by utilizing multiple surrogates. Moreover, the weighted average surrogates were never the worst surrogate in terms of optimization, and generally performed as well as the best individual surrogate for both sample sizes and both methods of generating the surrogate objective function.

The optimal fundamental blade frequencies presented in Tables 5 and 6 indicate that the optimal designs differ and that the BVI flight condition is characterized by many local optima. In order to illustrate the effectiveness of using multiple surrogates for identifying different low vibration designs, the predicted vibration reduction from each surrogate at all of the optimal designs from Table 6 are given in Table 7, along with predicted stress margins. While all surrogate objective functions predict that each optimal design is a reduced vibration design, some superior designs are missed due to errors in the surrogate constraints. For 
example, when using 283 sample points, the full term polynomial surrogate objective function predicts that the optimum design corresponding to the weighted average surrogate results in $484 \%$ reduction; which is superior to the $394 \%$ predicted reduction corresponding to its own optimum design. However, as indicated by the stress margin, the full term polynomial surrogate stress constraint incorrectly predicts that the weighted average optimum design yields. So if only full term polynomial surrogates were used, the superior design corresponding to the weighted average surrogates and $70.5 \%$ actual vibration reduction would not have been selected due to the inaccurate surrogate constraint. These results illustrate the benefits of utilizing multiple surrogates - for the relatively low cost of generating and optimizing multiple surrogates compared to the cost of generating the sample data, various low vibration designs were found which otherwise would be overlooked if only using a single approximation method. Furthermore, utilization of multiple surrogates was useful for locating various local optima in addition to the global optimum.

Table 7. Predicted vibration reduction by each of the surrogates at all of the optimal designs from Table 6 , along with predicted stress margins in parentheses.

\begin{tabular}{|c|c|c|c|c|c|c|c|}
\hline Optimum & $\begin{array}{c}\text { Sample } \\
\text { Size }\end{array}$ & $\begin{array}{c}\text { Predicted } \\
\text { Reduction \& } \\
\text { Stress Margin } \\
\text { w/ Poly. }\end{array}$ & $\begin{array}{c}\text { Predicted } \\
\text { Reduction \& } \\
\text { Stress Margin } \\
\text { w/ Poly(red) }\end{array}$ & $\begin{array}{c}\text { Predicted } \\
\text { Reduction \& } \\
\text { Stress Margin } \\
\text { w/ KRG }\end{array}$ & $\begin{array}{c}\text { Predicted } \\
\text { Reduction \& } \\
\text { Stress Margin } \\
\text { w/ RBNN }\end{array}$ & $\begin{array}{c}\text { Predicted } \\
\text { Reduction \& } \\
\text { Stress Margin } \\
\text { w/ Wtd. Avg. }\end{array}$ & $\begin{array}{c}\text { Predicted } \\
\text { Reduction \& } \\
\text { Stress Margin } \\
\text { w/ Wtd. Avg.(red) }\end{array}$ \\
\hline Poly. & 283 & $394 \%(0.0147)$ & $341 \%(0.0080)$ & $99 \%(0.0761)$ & $91 \%(0.0001)$ & $196 \%(0.0383)$ & $205 \%(0.0324)$ \\
\hline Poly(red) & 283 & $571 \%(-0.0002)$ & $512 \%(0.0001)$ & $115 \%(0.0083)$ & $94 \%(-0.0191)$ & $262 \%(-0.0003)$ & $287 \%(-0.0002)$ \\
\hline KRG & 283 & $298 \%(-0.0180)$ & $283 \%(-0.0098)$ & $120 \%(0.0840)$ & $91 \%(0.0005)$ & $173 \%(0.0298)$ & $187 \%(0.0275)$ \\
\hline RBNN & 283 & $427 \%(-0.0515)$ & $300 \%(-0.0482)$ & $90 \%(0.1141)$ & $94 \%(0.0001)$ & $203 \%(0.0295)$ & $184 \%(0.0207)$ \\
\hline Wtd. Avg. & 283 & $484 \%(-0.0263)$ & $417 \%(-0.0640)$ & $119 \%(0.0407)$ & $93 \%(0.0000)$ & $235 \%(0.0083)$ & $247 \%(-0.0130)$ \\
\hline Wtd. Avg.(red) & 283 & $387 \%(0.0464)$ & $378 \%(0.0435)$ & $99 \%(0.0620)$ & $92 \%(0.0002)$ & $193 \%(0.0436)$ & $222 \%(0.0427)$ \\
\hline Poly. & 484 & $222 \%(0.0076)$ & $200 \%(0.0077)$ & $72 \%(0.0074)$ & $93 \%(-0.0386)$ & $133 \%(-0.0007)$ & $130 \%(-0.0002)$ \\
\hline Poly(red) & 484 & $215 \%(0.0047)$ & $207 \%(0.0045)$ & $82 \%(0.0056)$ & $93 \%(-0.0379)$ & $135 \%(-0.0025)$ & $137 \%(-0.0022)$ \\
\hline KRG & 484 & $108 \%(0.0001)$ & $104 \%(0.0003)$ & $145 \%(0.0068)$ & $90 \%(-0.0426)$ & $120 \%(-0.0044)$ & $118 \%(-0.0043)$ \\
\hline RBNN & 484 & $48 \%(-0.0149)$ & $37 \%(-0.0012)$ & $104 \%(0.1076)$ & $97 \%(0.0004)$ & $81 \%(0.0380)$ & $75 \%(0.0410)$ \\
\hline Wtd. Avg. & 484 & $154 \%(-0.0286)$ & $146 \%(-0.0223)$ & $102 \%(0.0625)$ & $78 \%(0.0060)$ & $117 \%(0.0152)$ & $116 \%(0.0154)$ \\
\hline Wtd. Avg.(red) & 484 & $185 \%(-0.0002)$ & $185 \%(0.0005)$ & $86 \%(0.0045)$ & $91 \%(-0.0108)$ & $124 \%(-0.0001)$ & $129 \%(0.0002)$ \\
\hline
\end{tabular}

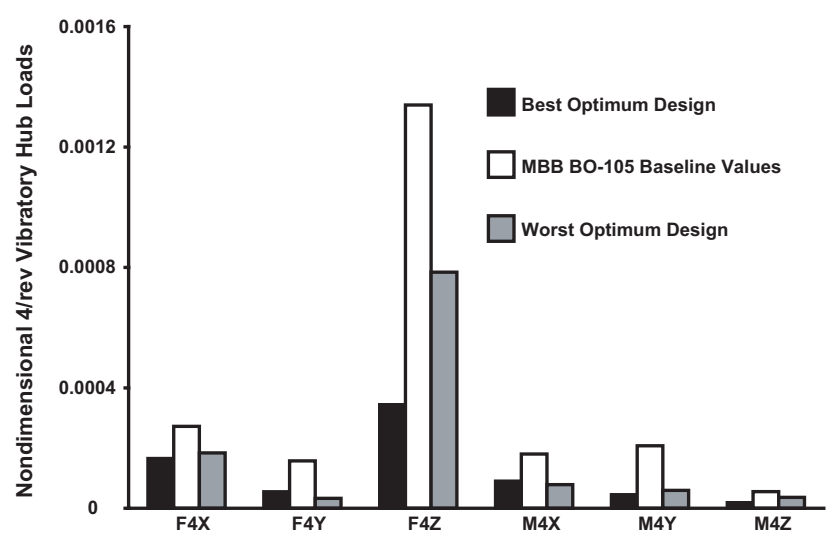

Figure 12. Comparison of the best and worst optimal designs with the baseline design.

are relatively close to the allowable stress.

The significant differences in predicted and actual amounts of vibration reduction in Tables 5 and 6 indicate that the surrogates are inaccurate at their respective optimal designs. Furthermore, the surrogates were susceptible to predicting impractical amounts of vibration reduction, i.e. $\geq 100 \%$. Thus it was especially critical for this problem to conduct simulations at each optimal design in order to obtain the actual amount of reduction. Even though they are not accurate every where in the design space, the surrogates still led to reduced vibration designs.

\section{VI.D. Global Sensitivity Analysis Results}

The variability in the surrogate objective function due to the most significant design variables are given in this section. All results in this section are based on direct approximation of the overall objective function. Figures 13 - 17 show the total variability including interactions among design variables and variability due 
to main effects. Design variables which are predicted to account for less than $3 \%$ of the total variability are considered to be relatively unimportant, and therefore are not shown. In addition, Figures 13 - 17 also give the total variability from all of the depicted design variables, as well as the total variability from the remaining design variables which individually account for less than $3 \%$ of the variance. Note that results generated with radial basis neural networks are not depicted because all design variables were predicted to account for $5-9 \%$ of the total variability. Thus, the GSA based on radial basis neural networks predicts that all of the design variables essentially have equal importance.

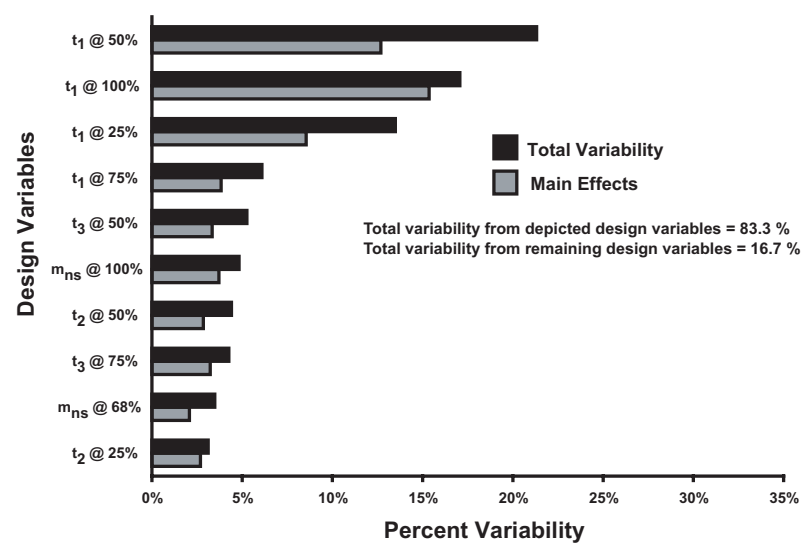

(a) Poly., 283 sample points

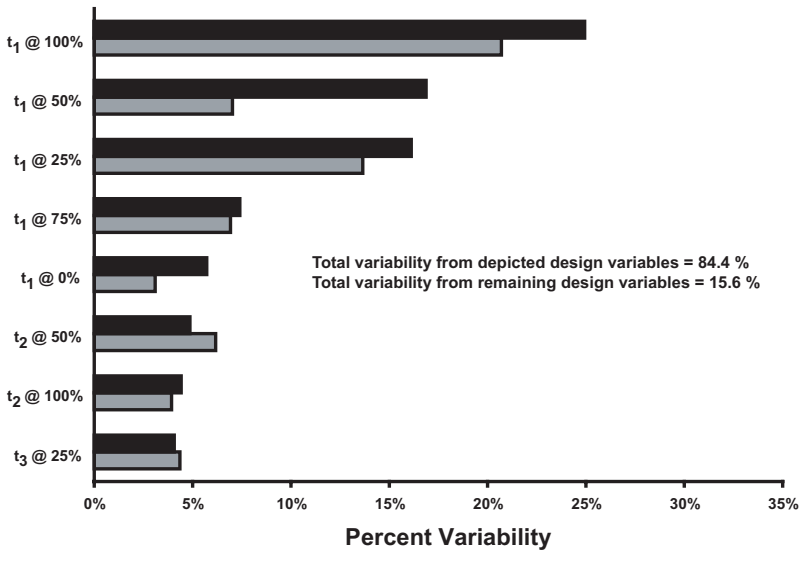

(b) Poly., 484 sample points

Figure 13. Contribution to the variability in the objective function from the most significant design variables using full term polynomial surrogates.

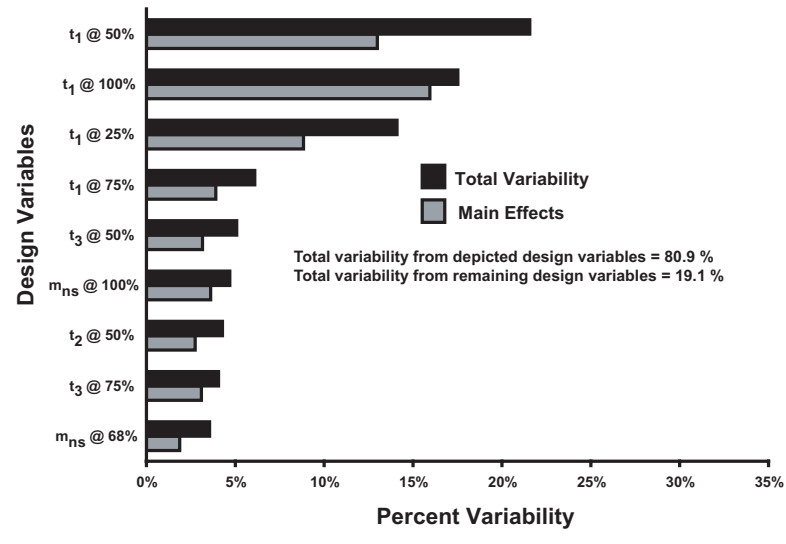

(a) Poly.(red), 283 sample points

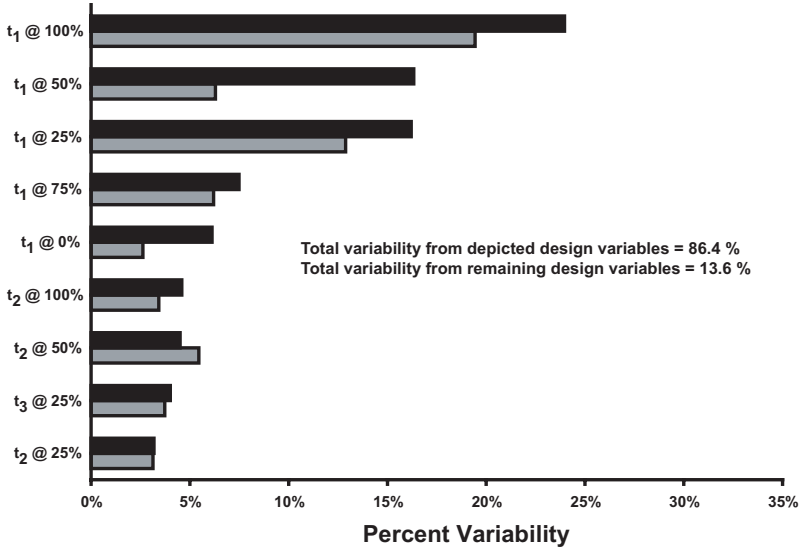

(b) Poly.(red), 484 sample points

Figure 14. Contribution to the variability in the objective function from the most significant design variables using reduced term polynomial surrogates.

From Figures 13 - 17 it is clear that the GSA predicts $t_{1}$ at the $25 \%, 50 \%$, and $100 \%$ blade stations are the three most important design variables, no matter what sample size or approximation method is used in the analysis. However, beyond this observation, the results of the global sensitivity analysis are highly dependent on the sample size and the approximation method. For instance, using the kriging surrogate and 283 sample points, $t_{1}$ at the $50 \%$ station is predicted to be the second most important variable. In contrast, this variable accounts for the third most variability in the objective function when using 484 sample points to create the surrogate. Furthermore, increasing the number of sample points changes which variables the GSA determines to be the most important for each surrogate. Comparing the results corresponding to the kriging surrogate with the other surrogates in Figures 13 - 17 with 283 sample points illustrates the effect of using different surrogates. The kriging surrogate predicts that 4 variables are the most significant and account for $89.5 \%$ of the total variability, while GSA with the other surrogates predicts that 7-10 design variables are significant. 


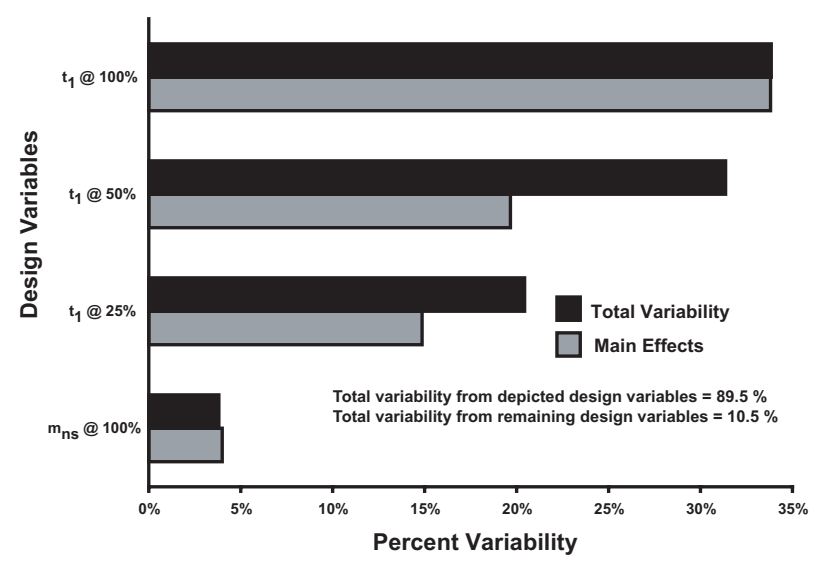

(a) KRG, 283 sample points

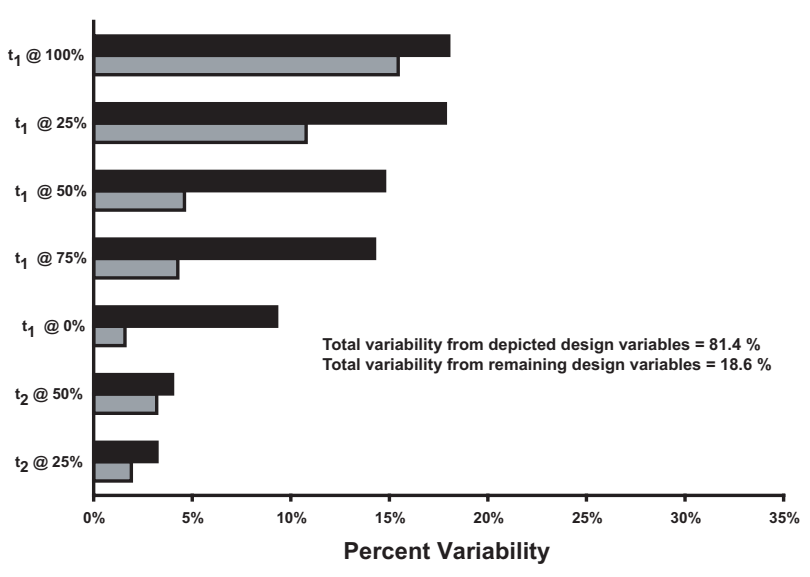

(b) KRG, 484 sample points

Figure 15. Contribution to the variability in the objective function from the most significant design variables using kriging surrogates.

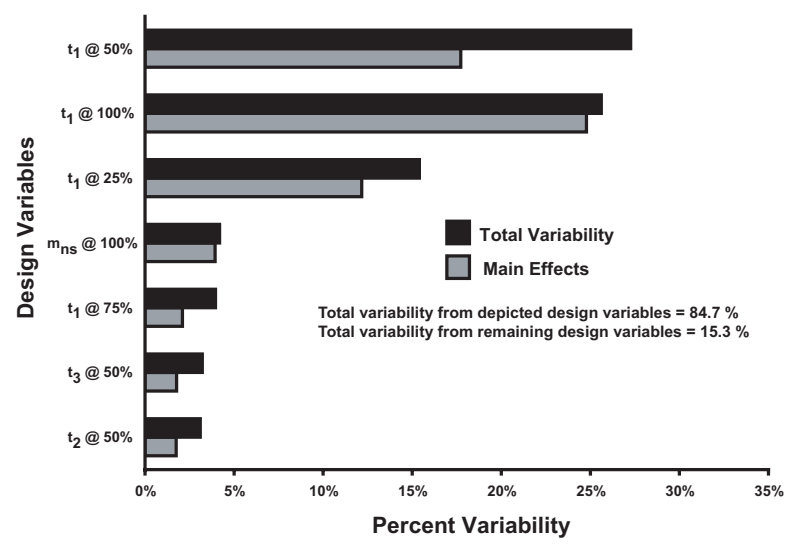

(a) Wtd. Avg., 283 sample points

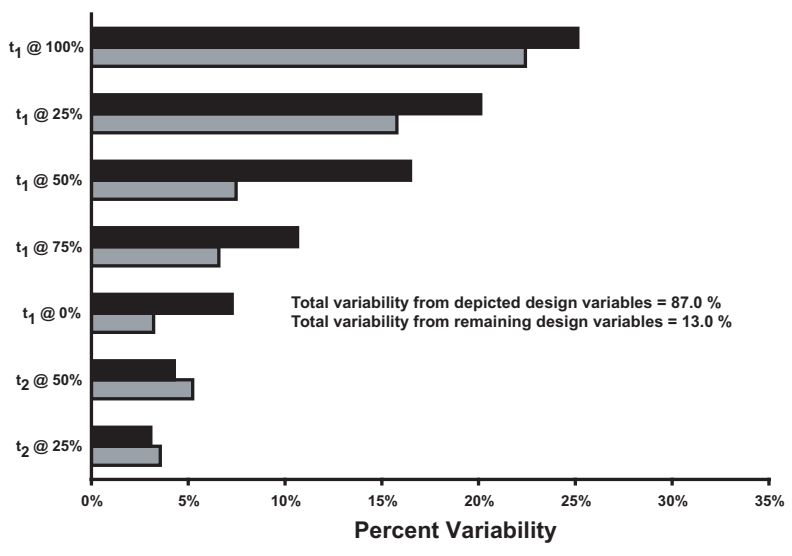

(b) Wtd. Avg., 484 sample points

Figure 16. Contribution to the variability in the objective function from the most significant design variables using weighted average surrogates with the full term polynomial.

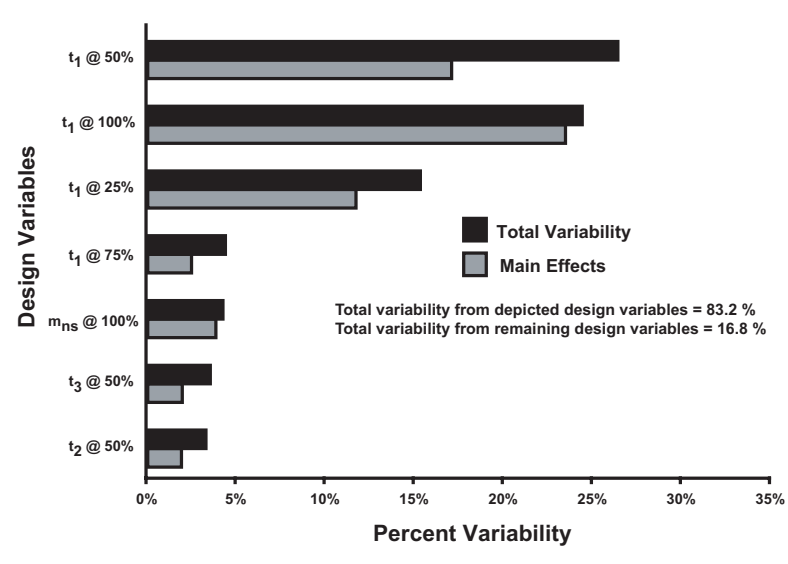

(a) Wtd. Avg.(red), 283 sample points

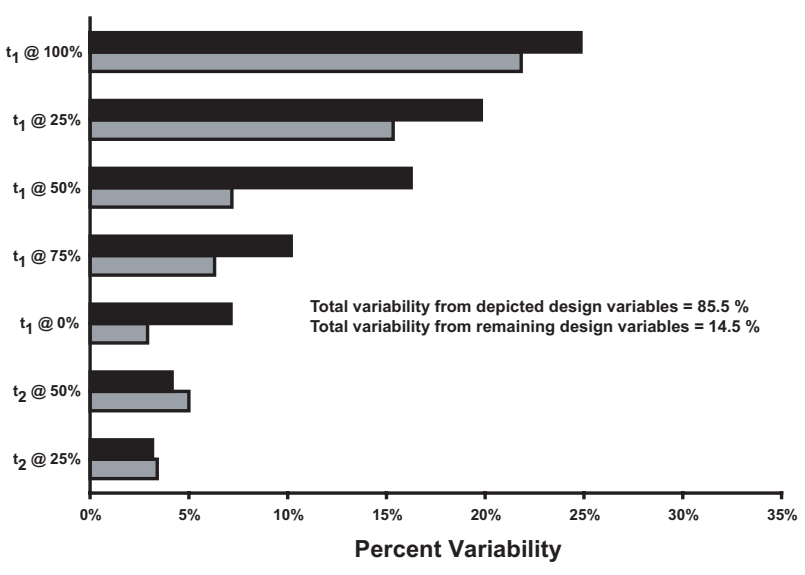

(b) Wtd. Avg.(red), 484 sample points

Figure 17. Contribution to the variability in the objective function from the most significant design variables using weighted average surrogates with the reduced term polynomial. 
In order to verify that the unimportant variables according to the GSA have little effect on the predictive capabilities of the surrogates, the surrogates were reconstructed after eliminating the unimportant design variables and the errors were compared to the errors when all of the design variables were included in the fitting process. The results of this comparison are summarized in Table 8, which gives the ratios of the reconstructed surrogates' errors to the errors obtained when using all of the design variables. The most extreme application of the GSA occurs in the case of kriging with 283 sample points, in which 13 of the 17 design variables were considered unimportant. In this case, the average error of the 4 dimensional kriging surrogate was $13.9 \%$ higher than the 17 dimensional kriging surrogate, while the maximum error was essentially the same. Other than the case of kriging with 283 sample points, the reconstructed surrogates after the GSA were only slightly less accurate or even more accurate - i.e. ratios less than 1 - than the original surrogates. For example, the reduced term polynomial based on 283 sample points is $21.3 \%$ more accurate than the original surrogate in terms of average error. Instances in which the reconstructed surrogates are more accurate suggest that the eliminated variables are unimportant since they impair the predictive capabilities of the surrogates when included.

Table 8. Ratio of errors after unimportant design variables are left out during surrogate construction to errors with all variables included

\begin{tabular}{ccccc}
\hline \hline $\begin{array}{c}\text { Surrogate } \\
\text { used in } \\
\text { GSA }\end{array}$ & $\begin{array}{c}\text { Sample } \\
\text { Size }\end{array}$ & $\begin{array}{c}\text { Number of } \\
\text { Eliminated } \\
\text { Variables }\end{array}$ & $\begin{array}{c}\text { Ratio of } \\
\text { Average } \\
\text { Errors }\end{array}$ & $\begin{array}{c}\text { Ratio of } \\
\text { Maximum } \\
\text { Errors }\end{array}$ \\
\hline Poly & 283 & 7 & 0.822 & 1.025 \\
Poly(red) & 283 & 8 & 0.787 & 0.971 \\
KRG & 283 & 13 & 1.139 & 1.023 \\
Wtd. Avg. & 283 & 10 & 1.030 & 0.878 \\
Wtd. Avg.(red) & 283 & 10 & 1.014 & 0.880 \\
\hline Poly & 484 & 9 & 0.917 & 0.980 \\
Poly(red) & 484 & 8 & 0.930 & 1.015 \\
KRG & 484 & 10 & 0.951 & 0.896 \\
Wtd. Avg. & 484 & 10 & 1.043 & 0.811 \\
Wtd. Avg.(red) & 484 & 10 & 1.048 & 0.838 \\
\hline \hline
\end{tabular}

The GSA was also utilized to refine the search for the optimum with each surrogate. After the unimportant design variables were removed, the reconstructed surrogates were optimized. The unimportant variables were fixed at their original optimum values from optimization when all variables were included in the surrogate construction. The results of this analysis are given in Table 9. Using the GSA to refine the optimization search generally resulted in superior designs to the original optimal designs. The most significant improvement occurred in the case of kriging surrogates based on 484 sample points, where the refined search led to an additional $13.8 \%$ vibration reduction. The weighted average surrogates were the only models in which the refined optimization did not always produce a better design. The failure of the refined search based on GSA to lead to better designs in the case of some of the weighted average surrogates does not necessarily mean that the GSA led to the removal of important design variables. Rather, the failure to produce a better design may be attributed to the fact that the surrogates are not accurate at the optimal designs.

\section{Conclusions}

The results in this paper demonstrate the effectiveness of employing multiple surrogates as a relatively inexpensive method for fully utilizing expensive sample data. In addition, it was shown that the weighted average surrogate approach protected against the worst individual surrogate and generally performed as well as the best in terms of predictive capability and optimization. It was demonstrated that when applying surrogate based optimization to the helicopter vibration reduction problem, superior blade designs may be overlooked if only a single approximation method is employed. Furthermore, the multiple individual surrogates can be combined into a weighted average surrogate whose performance was shown to be robust with respect to issues such as sample size and the various responses considered in this study. Finally, it was 
Table 9. Comparison of "actual" vibration reductions using reconstructed surrogates based on the GSA, with surrogates based on all design variables

\begin{tabular}{cccccccc}
\hline \hline Surrogate & $\begin{array}{c}\text { Sample } \\
\text { Size }\end{array}$ & $\begin{array}{c}\text { Actual } \\
\text { Reduction } \\
\text { (all variables) }\end{array}$ & $\begin{array}{c}\text { Actual } \\
\text { Reduction } \\
\text { (after GSA) }\end{array}$ & $\begin{array}{c}\text { Actual } \\
\text { Stress Margin } \\
\text { (after GSA) }\end{array}$ & $\boldsymbol{\omega}_{\boldsymbol{L} 1}$ & $\boldsymbol{\omega}_{\boldsymbol{F 1} 1}$ & $\boldsymbol{\omega}_{\boldsymbol{T} 1}$ \\
\hline Poly. & 283 & $64.4 \%$ & $70.4 \%$ & 0.005 & 0.623 & 1.058 & 4.521 \\
Poly(red) & 283 & $60.1 \%$ & $66.9 \%$ & 0.003 & 0.621 & 1.057 & 4.454 \\
KRG & 283 & $54.1 \%$ & $58.0 \%$ & 0.017 & 0.640 & 1.058 & 5.139 \\
Wtd. Avg. & 283 & $70.5 \%$ & $60.2 \%$ & 0.004 & 0.602 & 1.057 & 4.071 \\
Wtd. Avg.(red) & 283 & $65.0 \%$ & $64.8 \%$ & 0.003 & 0.613 & 1.059 & 3.952 \\
\hline Poly. & 484 & $45.0 \%$ & $52.4 \%$ & 0.006 & 0.626 & 1.057 & 4.346 \\
Poly(red) & 484 & $50.0 \%$ & $60.7 \%$ & 0.008 & 0.618 & 1.056 & 4.512 \\
KRG & 484 & $55.8 \%$ & $69.6 \%$ & 0.001 & 0.606 & 1.055 & 4.569 \\
Wtd. Avg. & 484 & $67.6 \%$ & $63.9 \%$ & 0.006 & 0.608 & 1.056 & 4.395 \\
Wtd. Avg.(red) & 484 & $58.8 \%$ & $65.1 \%$ & 0.004 & 0.616 & 1.056 & 4.497 \\
\hline \hline
\end{tabular}

demonstrated that the application of GSA to the helicopter vibration problem was an effective method for eliminating unimportant design variables. The principal results from this study are summarized below.

- The weighted average approach generally produced surrogates which were as effective as the "best" individual approximation method for predicting over the entire design space, regardless of the sample size. In contrast, the "best" individual surrogate for predicting over the entire design space was dependent on the sample size.

- The weighted average surrogates generally performed as well as any individual surrogate with respect to maximum errors, which are local error measures, even though a global error measure is used to construct the weighted average models.

- Regardless of whether the surrogate objective function was formed by approximating the underlying vibratory loads, or by directly approximating the overall response, the performance of the weighted average surrogates was comparable to the best individual method in terms of average error, and performed better than the worst approximation method in terms of maximum error.

- The weighted average surrogates were effective for locating reduced vibration designs for both sample sizes and both methods of generating the surrogate objective function.

- Optimization of multiple surrogates was an effective and relatively inexpensive method for locating reduced vibration blade designs which would have been overlooked if only a single surrogate was employed. Feasible designs ranging from $45-70.5 \%$ vibration reduction were located.

- The predictive capabilities of the surrogates could be enhanced by eliminating design variables which are determined to be unimportant using GSA. In the instances where the predictive capability was not enhanced, the error was generally only slightly higher.

- It is possible to locate superior blade designs by refining the search for the optimum based on the GSA. However, if the surrogates are not accurate at the optimal designs, the refined search may not always lead to improved blade designs.

\section{Appendix: Generalized Mean Square Cross-Validation Error}

In general, the data is divided into $k$ subsets, i.e. $k$-fold cross-validation, of approximately equal size. A surrogate model is constructed $k$ times, each time leaving out one of the subsets from training, and using the omitted subset to compute the error measure of interest. The generalization error estimate is computed using the $k$ error measures obtained (e.g., average). If $k$ equals the number of sample points, this 
approach is called leave-one-out cross-validation (also known as PRESS in the polynomial response surface approximation terminology). Equation (53) represents a leave-one-out calculation when the generalization error is described by the mean square error,

$$
G M S E_{i}=\frac{1}{k} \sum_{i=1}^{k}\left(y_{i}-\hat{y}_{i}^{(-i)}\right)^{2}
$$

where $\hat{y}_{i}^{(-i)}$ represents the prediction at $\mathbf{x}^{(i)}$ using the surrogate constructed with all sample points except $\left(\mathbf{x}^{(i)}, y_{i}\right)$. The advantage of cross-validation is that it provides a nearly unbiased estimate of the generalized error and the corresponding variance is reduced, when compared to split-sample, considering that every point gets to be in a test set once, and in a training set $(k-1)$ times, regardless of how the data is divided.

\section{Acknowledgments}

This research was supported in part by the FXB Center for Rotary and Fixed Wing Air Vehicle Design, NASA GSRP grant NNA-06CB71H for Bryan Glaz, and partial funding from the Center for Rotorcraft Innovation (CRI) under WBS 2006-B-01-01.2-A17. Support for Tushar Goel was provided by the Institute for Future Space Transport, under the NASA Constellation University Institute Program (CUIP), with Ms. Claudia Meyer as program monitor. Furthermore, some of the material in this study is based upon work supported by the National Science Foundation under Grant No. 0423280.

\section{References}

\footnotetext{
${ }^{1}$ Friedmann, P. P., "Helicopter Vibration Reduction Using Structural Optimization with Aeroelastic/Multidisciplinary Constraints - A Survey," Journal of Aircraft, Vol. 28, No. 1, January 1991, pp. 8-21.

${ }^{2}$ Celi, R., "Recent Applications of Design Optimization to Rotorcraft-A Survey," Journal of Aircraft, Vol. 36, No. 1, January-February 1999, pp. 176-189.

${ }^{3}$ Ganguli, R., "Survey of Recent Developments in Rotorcraft Design Optimization," Journal of Aircraft, Vol. 41, No. 3, May-June 2004, pp. 493-510.

${ }^{4}$ Friedmann, P. P. and Millott, T. A., "Vibration Reduction in Rotorcraft Using Active Control: A Comparison of Various Approaches," Journal of Guidance, Control, and Dynamics, Vol. 18, No. 4, July-August 1995, pp. 664-673.

${ }^{5}$ Friedmann, P. P., "Rotary Wing Aeroelasticity - Current Status and Future Trends," AIAA Journal, Vol. 42, No. 10, October 2004, pp. 1953-1972.

${ }^{6}$ Queipo, N. V., Haftka, R. T., Shy, W., Goel, T., Vaidyanathan, R., and Tucker, P. K., "Surrogate-Based Analysis and Optimization," Progress in Aerospace Sciences, Vol. 41, 2005, pp. 1-28.

${ }^{7}$ Won, K. and Ray, T., "A Framework for Design Optimization using Surrogates," Engineering Optimization, Vol. 37, No. 7, October 2005, pp. 685-703.

${ }^{8}$ Simpson, T. W., Booker, A. J., Ghosh, D., Giunta, A. A., Koch, P. N., and Yang, R., "Approximation Methods in Multidisciplinary Analysis and Optimization: A Panel Discussion," Structural and Multidisciplinary Optimization, Vol. 27, No. 5, July 2004, pp. 302-313.

${ }^{9}$ Glaz, B., Friedmann, P. P., and Liu, L., "Surrogate Based Optimization of Helicopter Rotor Blades for Vibration Reduction in Forward Flight," 47th AIAA/ASME/ASCHE/AHS/ASC Structures, Structural Dynamics E Materials Conference, Newport, RI, May 1-4 2006, pp. 1-21, AIAA Paper 2006-1821.

${ }^{10}$ Goel, T., Haftka, R. T., Shyy, W., and Queipo, N. V., "Ensemble of Surrogates," Structural and Multidisciplinary Optimization, Vol. 33, 2007, pp. 199-216.

${ }^{11}$ Zerpa, L., Queipo, N., Pintos, S., and Salager, J., "An Optimization Methodology of Alkaline-Surfactant-Polymer Flooding Process using Field Scale Numerical Simulation and Multiple Surrogates," Journal of Petroleum Science and Engineering, Vol. 47, 2005, pp. 197-208.

${ }^{12}$ Goel, T., Zhao, J., Thakur, S. S., Haftka, R. T., and Shyy, W., "Surrogate Model-Based Strategy for Cryogenic Cavitation Model Validation and Sensitivity Evaluation," 42nd AIAA/ASME/SAE/ASEE Joint Propulsion Conference and Exhibit, Sacramento, CA, July 9-12 2006, AIAA Paper 2006-5047.

${ }^{13}$ Samad, A., Kim, K. Y., Goel, T., Haftka, R. T., and Shyy, W., "Shape Optimization of Turbomachinery Blade using Multiple Surrogate Models," ASME Joint US-European Fluids Engineering Division Summer Meeting, Miami, FL, July 17-20 2006, FEDSM 2006-98368.

${ }^{14}$ Sobol, I., "Sensitivity Analysis for Nonlinear Mathematical Models," Mathematical Modeling $\&$ Computational Experiment, Vol. 1, No. 4, 1993, pp. 407-414.

${ }^{15}$ Vaidyanathan, R., Goel, T., Shyy, W., and Haftka, R. T., "Global Sensitivity and Trade-Off Analysis for Multi-Objective Liquid Rocket Injector Design," 40th AIAA/ASME/SAE/ASEE Joint Propulsion Conference and Exhibit, Ft. Lauderdale, FL, July 11-14 2004, AIAA 2004-4007.

${ }^{16}$ Mack, Y., Goel, T., Shyy, W., and Haftka, R. T., "Multiple Surrogates for the Shape Optimization of Bluff BodyFacilitated Mixing," 43rd AIAA Aerospace Sciences Meeting and Exhibit, Reno, NV, January 10-13 2005, AIAA $2005-0333$.
} 
${ }^{17}$ Homma, T. and Saltelli, A., "Importance Measures in Global Sensitivity Analysis of Nonlinear Models," Reliability Engineering and Systems Safety, Vol. 52, 1996, pp. 1-17.

${ }^{18}$ Millott, T. A. and Friedmann, P. P., Vibration Reduction in Helicopter Rotors Using an Actively Controlled Partial Span Trailing Edge Flap Located on the Blade, NASA CR 4611, June 1994.

${ }^{19}$ Yuan, K. A. and Friedmann, P. P., Aeroelasticity and Structural Optimization of Composite Helicopter Rotor Blades with Swept Tips, NASA CR 4665, May 1995.

${ }^{20}$ Myrtle, T. F. and Friedmann, P. P., "Application of a New Compressible Time Domain Aerodynamic Model to Vibration Reduction in Helicopters Using an Actively Controlled Flap," Journal of the American Helicopter Society, Vol. 46, No. 1, Jan. 2001, pp. 32-43.

${ }^{21}$ de Terlizzi, M. and Friedmann, P. P., "Active Control of BVI Induced Vibrations Using a Refined Aerodynamic Model and Experimental Correlation," Proceedings of the 55th Annual Forum of the American Helicopter Society, Montreal, Canada, May 1999, pp. 599-615.

${ }^{22}$ Depailler, G. and Friedmann, P. P., "Reductions of Vibrations Due to Dynamic Stall in Helicopters Using an Actively Controlled Flap," Proceedings of the 43rd AIAA/ASME/ASCE/AHS/ACS Structures, Structural Dynamics and Materials Conference, Denver, CO, April 2002, AIAA Paper No. 2002-1431.

${ }^{23}$ Patt, D., Liu, L., and Friedmann, P. P., "Simultaneous Vibration and Noise Reduction in Rotorcraft Using Aeroelastic Simulation," Journal of the American Helicopter Society, Vol. 51, No. 2, 2006, pp. 127-140.

${ }^{24}$ Liu, L., Friedmann, P. P., and Patt, D., "Simultaneous Vibration and Noise Reduction in Rotorcraft - Practical Implementation Issues," Proceedings of the 46th AIAA/ASME/ASCE/AHS/ACS Structures, Structural Dynamics and Materials Conference, Austin, TX, April 2005, AIAA Paper 2005-2245.

${ }^{25}$ Yuan, K. A. and Friedmann, P. P., "Structural Optimization for Vibratory Loads Reduction of Composite Helicopter Rotor Blades with Advanced Geometry Tips," Journal of the American Helicopter Society, Vol. 43, No. 3, July 1998, pp. 246256.

${ }^{26}$ Myrtle, T. F., Development of an Improved Aeroelastic Model for the Investigation of Vibration Reduction in Helicopter Rotors using Trailing Edge Flaps, Ph.D. thesis, University of California, Los Angeles, 1998, Mechanical and Aerospace Engineering.

${ }^{27}$ Patt, D., Liu, L., and Friedmann, P. P., "Rotorcraft Vibration Reduction and Noise Prediction Using a Unified Aeroelastic Response Simulation," Journal of the American Helicopter Society, Vol. 50, No. 1, January 2005, pp. 95-106.

${ }^{28}$ Johnson, W., CAMRAD/JA - A Comprehensive Analytical Model of Rotorcraft Aerodynamics and Dynamics, Vol I. Theory Manual, Johnson Aeronautics, Palo Alto, CA, 1988.

${ }^{29}$ Johnson, W., CAMRAD/JA - A Comprehensive Analytical Model of Rotorcraft Aerodynamics and Dynamics, Vol II. Users' Manual, Johnson Aeronautics, Palo Alto, CA, 1988.

${ }^{30}$ de Terlizzi, M. and Friedmann, P. P., "Aeroelastic Response of Swept Tip Rotors Including the Effects of BVI," Proceedings of the 54th Annual Forum of the American Helicopter Society, Washington D.C., May 1998, pp. 644-663.

${ }^{31}$ Friedmann, P. P. and Shanthakumaran, P., "Optimum Design of Rotor Blades for Vibration Reduction in Forward Flight," Journal of the American Helicopter Society, Vol. 29, No. 4, 1984, pp. 70-80.

${ }^{32} \mathrm{Lim}, \mathrm{J}$. W. and Chopra, I., "Aeroelastic Optimization of a Helicopter Rotor," Journal of the American Helicopter Society, Vol. 34, No. 1, 1989, pp. 55-62.

${ }^{33}$ Celi, R. and Friedmann, P. P., "Structural Optimization with Aeroelastic Constraints of Rotor Blades with Straight and Swept Tips," AIAA Journal, Vol. 28, No. 5, 1990, pp. 928-936.

${ }^{34}$ Sacks, J., Welch, W. J., Mitchell, T. J., and Wynn, H. P., "Design and Analysis of Computer Experiments," Statistical Science, Vol. 4, No. 4, 1989, pp. 409-435.

${ }^{35}$ McKay, M. D., Beckman, R. J., and Conover, W. J., "A Comparison of Three Methods for Selecting Values of Input Variables in the Analysis of Output from a Computer Code," Technometrics, Vol. 21, No. 2, May 1979, pp. $239-245$.

${ }^{36}$ Jin, R., Chen, W., and Sudjianto, A., "An Efficient Algorithm for Constructing Optimal Design of Computer Experiments," Journal of Statistical Planning and Inference, Vol. 134, No. 1, 2005, pp. 268-287.

${ }^{37}$ Koch, P. N., Evans, J. P., and Powell, D., "Interdigitation for Effective Design Space Exploration using iSIGHT," Structural and Multidisciplinary Optimization, Vol. 23, No. 2, 2002, pp. 111-126.

${ }^{38}$ Jin, R., Chen, W., and Simpson, T. W., "Comparative Studies of Metamodeling Techniques Under Multiple Modeling Criteria," Structural and Multidisciplinary Optimization, Vol. 23, 2001, pp. 1-13.

${ }^{39}$ Jones, D. R., Schonlau, M., and Welch, W. J., "Efficient Global Optimization of Expensive Black-Box Functions," Journal of Global Optimization, Vol. 13, 1998, pp. 455-492.

${ }^{40}$ Sasena, M., Flexibility and Efficiency Enhancements for Constrained Global Optimization with Kriging Approximations, Ph.D. thesis, University of Michigan, 2002, Mechanical Engineering.

${ }^{41}$ Simpson, T. W., Peplinski, D., Koch, P. N., and Allen, J. K., "Metamodels for Computer-based Engineering Design: Survey and recommendations," Engineering with Computers, Vol. 17, 2001, pp. 129-150. 